\title{
Spatial variability of Brunic Arenosols and associated soils along the slope of the Słupia River valley (middle Pomerania, northern Poland)
}

\author{
Jerzy Jonczak ${ }^{1 *}$, Krzysztof Sztabkowski $^{2}$ \\ ${ }^{1}$ Warsaw University of Life Sciences, Institute of Agriculture, Department of Soil Science, Nowoursynowska Str. 159, 02-776 Warsaw, Poland \\ ${ }^{2}$ Forest Research Institute, Sękocin Stary, Braci Leśnej Str. 3, 05-090 Raszyn, Poland \\ * Dr hab. J. Jonczak, jerzy_jonczak@sggw.edu.pl, ORCID iD: https://orcid.org/0000-0002-9577-5292
}

\author{
Received: 23.06.2021 \\ Accepted: 11.11.2021 \\ Associated editor: J. Waroszewski
}

\section{Keywords}

Brunic Arenosols

Soil formation

Slope processes

Fluvioglacial landscape

Soil properties

\begin{abstract}
This study was aimed at evaluating the spatial heterogeneity of the soil cover along the slope of the Słupia River valley (Middle Pomerania, northern Poland). Various soil properties are discussed in a context of parent material origin and its transformation through post-depositional processes, and the role of relief as a factor influencing the spatial variability of the soils and sediments. A broad spectrum of soil characteristics, including texture, contents of elements and indicators of soil ecochemical state, weathering and pedogenesis, were determined from 10 soil profiles using standard procedures. Brunic Arenosols were a major component of the studied sequences. However, their morphology varied, reflecting the impact of the post-depositional transformation of their parent materials by water erosion and other processes typical for slopes. The Ti:Zr ratio suggested only a local-scale transformation of the primary deposits. Typically, soils from the upper slope locations and the transitional zone between the valley slope and the supra-flood terrace were characterised by lower solum thicknesses when compared to the slope foot, middle slope and valley bottom. The presence of colluvial horizons in some locations constituted evidence downslope sediment transport. Weathering features in the studied soils were evidenced by the presence of brown or rust coloured B horizons enriched in pedogenic forms of Fe and Al. The distribution of free Fe and Al along the studied slope suggested lateral transport of those elements. The soils strongly varied in terms of total organic $\mathrm{C}$ and $\mathrm{N}$ contents and stocks, the highest stocks of these being noted in soils from higher locations and the slope foot, and being the lowest in mid-slope locations. The $\mathrm{P}, \mathrm{K}, \mathrm{Ca}$ and $\mathrm{Mg}$ contents were typically low in these sandy soils. All the soils were characterised by typical $\mathrm{pH}$ and sorptive characteristics, although their spatial variability was considerable.
\end{abstract}

\section{Introduction}

Brunic Arenosols constitute one of the major components of soil cover in the temperate climatic zone. Sandy materials of glacial, fluvioglacial and aeolian origin, as well as the products of weathering of some certain rock types are typical substrates in their formation. The most characteristic feature of these soils is their relatively simple structure, with a rust-coloured B horizon (Bv, sideric) enriched in free sesquioxides, resulting from weathering and biogenic accumulation. Although the origin and properties of Brunic Arenosols have been the subject of many studies, there is no consensus among soil scientists on many of their aspects. The role of a periglacial environment in the formation of the Bv horizon and the age of the soils are the issues most discussed. Some authors (e.g. Kowalkowski and Nowak, 1968; Kowalkowski, 1976, 1977a; Manikowska, 1997) have highlighted the importance of frost weathering and the periglacial origin of Brunic Arenosols. However, r Prusinkiewicz (1965), Banaszuk
(1977), Bednarek (1991) and Janowska (2001), among other authors, have found evidence of a Holocene origin and a great importance of vegetation in their formation. Degórski (2002) found correlations between parent material age (depositional period), climatic conditions and associated vegetation and certain characteristics of Brunic Arenosols. However, Bednarek (1991) and Janowska $(1994,2001)$ did not observe any correlation between the chemical properties of the Bv horizon and soil age.

Currently, Brunic Arenosols are mainly forest soils. They are most often covered by coniferous species, although some studies have indicated that they are also associated with deciduous forests (Ferczyńska-Uggla, 1976; Andrzejczyk and Sewerniak, 2016). Biały (1999) reported that, due to the high susceptibility of Brunic Arenosols to podzolisation, the use of coniferous species should be limited in forests. Błońska et al. (2013) demonstrated great variability in these soils in terms of enzymatic activity, which is strongly related to soil sub-type and vegetation richness. Forest Brunic Arenosols have been majorly affected by direct and 
indirect human activity in both past and modern times. Anthropogenic changes have affected the morphology and chemistry of the soils due to agricultural use (Czubaszek and Banaszuk, 2004; Dłużewski et al., 2019), deforestation and forest site preparation (Sewerniak et al., 2014), forest management practices (Łabęda and Kondras, 2020), military activities (Jankowski, 2019), charcoal production (Hirsch et al., 2018) and wildfires (Gonet, 2010), among many other things. The most commonly observed podsolisation is an effect of the introduction of coniferous monocultures into forests replacing the natural vegetation (Jankowski, 2014a). In this context, the importance of soil model areas, as refuges for close to natural Brunic Arenosols should be highlighted (Chojnicki et al., 2021).

The issue of the position in the landscape of Brunic Arenosols and their spatio-temporal linkage with other components of terrestrial ecosystems, in particular, other soils is of great interest, although still poorly explored. Brunic Arenosols occur in association with Podzols, Cambisols, Luvisols, Phaeozems and other soil units. The transition between Brunic Arenosols and other components of the soil cover is smooth, with sometimes only quantitative criteria allowing distinction between the soil reference groups (Janowska, 2001). Considering the importance of Brunic Arenosols in the temperate climatic zone, the dynamic changes that can be observed over the last few centuries as a result of various aspects of human activity and their stratigraphic position, further studies are highly recommended.
This study aimed to evaluate the spatial variability of Brunic Arenosols and associated soils along the slope of the Słupia River valley located in the landscape of Middle Pomerania (northern Poland). The various soil characteristics are discussed in a context of the origin of the parent material and its transformation through post-depositional processes, and the role of relief as a factor influencing the spatial heterogenity of the soils and sediments. The study covered a broad spectrum of soil characteristics, including texture, elemental content and indicators of soil eco-chemical state, weathering and pedogenesis.

\section{Material and methods}

The study was performed in northern Poland, south of Słupsk City (Fig. 1). The landscape of the studied area was developed by ice sheet transgression during the Pomeranian Phase of the Vistula Glaciation, followed by geomorphological processes in the periglacial zone during the Gardno Phase of the Vistula glaciation in addition to Holocene processes (Florek, 1991; Kozarski, 1995). The contemporary climate is relatively mild due to the influence of the Baltic Sea. Mean annual temperatures for the period 1950-2007 varied from 6.0 to $9.7^{\circ} \mathrm{C}$ (mean $7.8^{\circ} \mathrm{C}$ ) whereas the annual sum of precipitation is from 521.7 to $1260.5 \mathrm{~mm}$ (mean $793.8 \mathrm{~mm}$ ) (Kirschenstein and Baranowski, 2008). The study involved 10 soil profiles
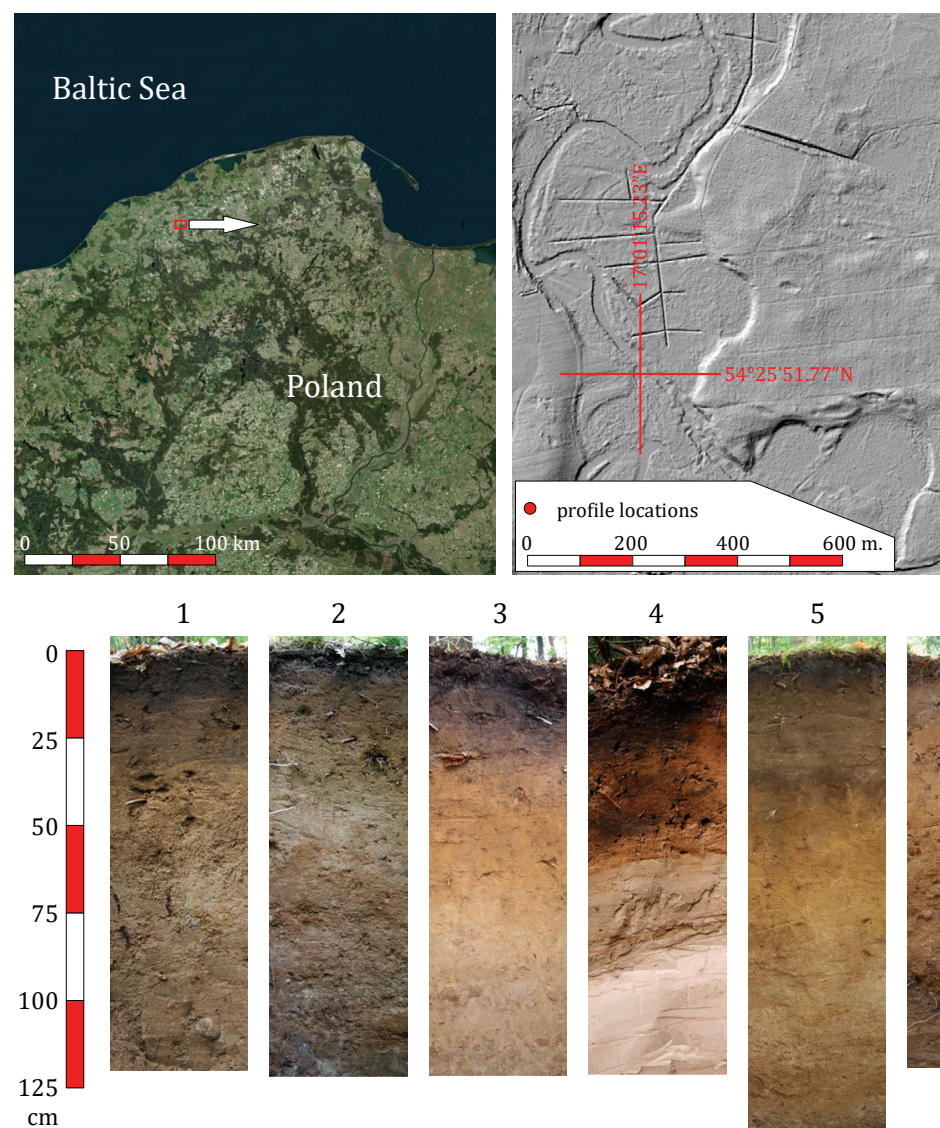

4
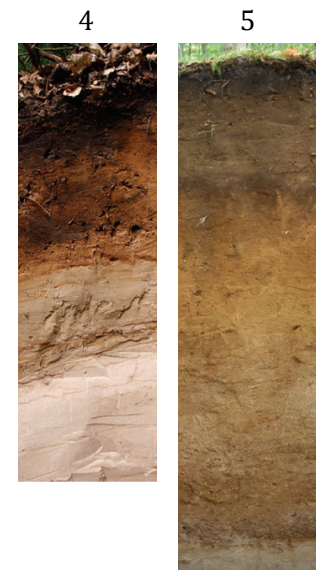

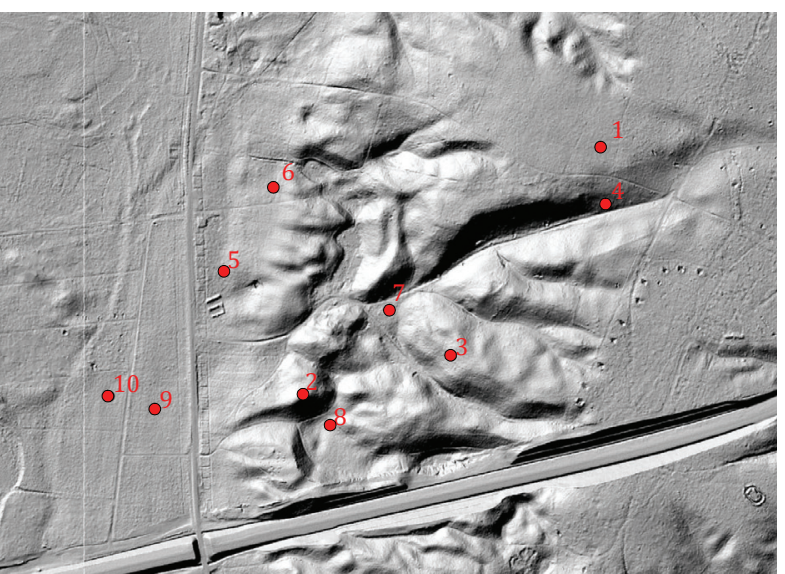

6



7

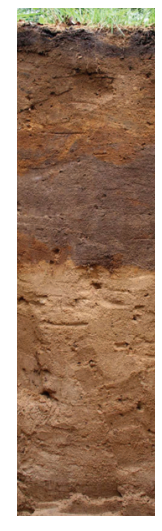

8

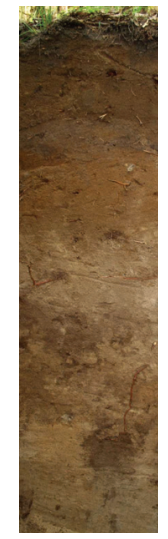

9

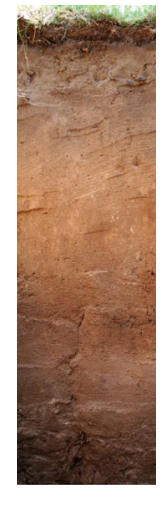

10



Fig. 1. Locations and morphology of the studied soils 
distributed along a toposequence of the west-facing slope of the Słupia River valley. The slope was strongly affected by water erosion, which is reflected in its morphology (Fig. 1). The soil sequence covered the marginal part of the plateau (location 1), built-up of glacial till covered with a thin layer of fluvioglacial sand; dissected land elevations covered with residual deposits over glacial till (location 2); several slope locations within fluvioglacial sandy cover (locations 3-6); the bottom parts of a dry valley partially filled with colluvial deposits over fluvioglacial sand (locations 7 and 8) and the transitional zone between the river valley slope and the supra-flood terrace (locations 9 and 10) (Fig. 1, Table 1). The area is currently afforested, with Scots pine predominating in the tree layer.

One soil pit was done at each location. The soils were described using the Food and Agriculture Organization of the United Nations (FAO, 2006) criteria, classified according to the World Reference Base (WRB) system (IUSS Working Group WRB, 2015) and then sampled. One disturbed sample and two undisturbed $100 \mathrm{~cm}^{3}$ samples were taken from each soil horizon. Undisturbed samples were dried at $105^{\circ} \mathrm{C}$ and weighted. The disturbed samples were air-dried and sieved through a $2.0 \mathrm{~mm}$ sieve to remove the gravel fraction. Analyse of the earth fraction included:

- $\quad$ Particle-size distribution using the mixed pipette and sieve methods. Polish Soil Science Society (PTG 2009) classification of textural fractions and groups was applied; Bulk density of the undisturbed samples using the gravimetric method;

- $\quad \mathrm{pH}$ in a suspension with water and $1 \mathrm{~mol} \mathrm{dm}^{-3} \mathrm{KCl}$ solution at a soil:water/KCl ratio of $1: 2.5$ for the mineral samples and 1:10 for the organic samples using the potentiometric method;

- Total organic carbon (TOC) and nitrogen (N) contents by dry combustion (Vario MacroCube, Elementar Germany);

- Total contents of P, K, Ca, Mg, Fe, Al, Ti and $\mathrm{Zr}$ by inductively coupled plasma atomic emission spectrometry (ICP-OES, Avio 200, Perkin Elmer) after samples digestion in a mixture of $40 \% \mathrm{HF}, 65 \% \mathrm{HNO}_{3}$ and $38 \% \mathrm{HCl}$ in the proportion $5: 3: 2$ by volume;

- The contents of free $\mathrm{Fe}\left(\mathrm{Fe}_{\mathrm{d}}\right)$ using the Mehra and Jackson (1960) extraction procedure, and amorphous $\mathrm{Fe}\left(\mathrm{Fe}_{0}\right)$ and $\mathrm{Al}$ $\left(\mathrm{Al}_{\mathrm{o}}\right.$ ) following extraction using the Schwertmann method (Van Reeuvijk, 1995). The elemental contents of the extracts were determined by ICP-OES;

- Exchangeable acidity $\left(\mathrm{H}_{\mathrm{w}}\right)$ and the exchangeable $\mathrm{Al}\left(\mathrm{Al}_{\mathrm{w}}\right)$ content using the Sokolov method, and the content of exchangeable bases $\left(\mathrm{Ca}^{2+}, \mathrm{Mg}^{2+}, \mathrm{K}^{+}\right.$and $\left.\mathrm{Na}^{+}\right)$using ICP-OES, after sample extraction in a $1 \mathrm{~mol} \mathrm{dm}^{-3}$ solution of ammonium acetate, $\mathrm{pH}=7.0$. Based on the results, the sum of exchangeable bases (TEB) $\left(\mathrm{Ca}^{2+}+\mathrm{Mg}^{2+}+\mathrm{K}^{+}+\mathrm{Na}^{+}\right)$, the cation exchange capacity (CEC) $\left(\mathrm{H}_{\mathrm{w}}+\mathrm{TEB}\right)$ and the effective saturation of the soil sorption complex with basic cations ((TEB $\cdot 100 \%) /\left(\mathrm{Ca}^{2+}\right.$ $\left.+\mathrm{Mg}^{2+}+\mathrm{K}^{+}+\mathrm{Na}^{+}+\mathrm{Al}_{\mathrm{w}}\right)$ ) were calculated.

The statistical analysis included obtaining the correlation coefficients and performing a cluster analysis. The analysis was performed using Past 3.0 software. Quantum GIS 2.18 was used for spatial visualisation of the results.

\section{Results}

\subsection{Soil classification, morphology and basic physical characteristics}

Eutric Umbric Planosols, developed from a thin cover of fluvioglacial sand over glacial till, were typical of the marginal zone of the plateau (Profile 1). Dystric Brunic Arenosols (Profiles 2, 3, 4, 6 and 9) and Dystric Albic Brunic Arenosols (Profile 10) constituted major components of eroded land elevations and slopes built from fluvioglacial and residual sands, whereas Brunic Umbrisols (Profile 5), Brunic Umbrisols (Colluvic) (Profile 8) and Dystric Arenosols (Colluvic) (Profile 7) occured at lower locations. The morphology of the soils varied along the slope, reflecting soil-forming processes, the predominant role of

Table 1

Basic characteristics of the studied profile locations

\begin{tabular}{llll}
\hline Profile number & Location in relief & $\begin{array}{l}\text { Dominant (admixtures) } \\
\text { species in tree layer* }\end{array}$ & Origin of parent material \\
\hline 1 & Plateau & B (O) & Fluvioglacial sand over glacial till \\
2 & Plateau & O & Residual sand over glacial till \\
3 & Upper slope & P, B & Fluvioglacial sand \\
4 & Middle slope & B (O,S) & Fluvioglacial sand \\
5 & Foot slope & S,P (B) & Fluvioglacial sand \\
6 & Foot slope & P (B) & Fluvioglacial sand \\
7 & Dry valley bottom & O & Colluvium over fluvioglacial sand \\
8 & Dry valley botton & O (M) & Colluvium over fluvioglacial sand \\
9 & Fluvioglacial plain & P (O,H) & Fluvioglacial sand \\
10 & Fluvioglacial plain & P (SB,O) & Fluvioglacial sand \\
\hline
\end{tabular}

P - Scots pine, SB - Siver birch, O - Pedunculated oak, H - European hornbeam, B - European beech, S - Norway spruce, M - Common maple 
various factors of soil formation and the spatial linkage between the components of the soil cover (Fig. 1). The solum depth varied from 44 to $120 \mathrm{~cm}$, including transitional zones to the parent material and buried horizons. The thickness of the A horizons in the studied soils varied from 7 to $37 \mathrm{~cm}$. This depth was the shallowest in the upper and mid-slope locations and the deepest at the slope foot and in the dry valley bottom. The A horizons in profiles 1, 5, 7 and 10 were characterised by a vertical heterogeneity. Weathering features occured in the soils as rust-coloured horizons (Bw), or a brownish-coloured upper part of the luvic horizon (EtBw) in profile 1. The morphology of the Bw horizons varied greatly, including the thickness, colour and transition into the $\mathrm{C}$ horizon (Table 2). In profiles 3, 4, 6, 7 and 9 the there was an initial podsolisation and/or there were lithological

Table 2

Particle-size distribution and basic physical characteristics of the studied soils

\begin{tabular}{|c|c|c|c|c|c|c|c|c|}
\hline Horizon & $\begin{array}{l}\text { Depth } \\
\text { [cm] }\end{array}$ & $\begin{array}{l}\text { Gravel } \\
\%\end{array}$ & $\begin{array}{l}\text { Sand } \\
\%\end{array}$ & $\begin{array}{l}\text { Silt } \\
\%\end{array}$ & $\begin{array}{l}\text { Clay } \\
\%\end{array}$ & Texture* & $\begin{array}{l}\text { Soil } \\
\text { colour }\end{array}$ & $\begin{array}{l}\text { Bulk } \\
\text { density } \\
\mathrm{g} \mathrm{cm}^{-3}\end{array}$ \\
\hline \multicolumn{9}{|c|}{ Profile 1 - Eutric Umbric Planosol } \\
\hline A & $0-10$ & 1.8 & 80.6 & 14.2 & 5.3 & ls & 10YR $2 / 2$ & 0.88 \\
\hline$A(p)$ & $10-28$ & 3.6 & 87.3 & 11.3 & 1.4 & ls & 10YR 2.5/3 & 1.45 \\
\hline EtBw & $28-48$ & 3.2 & 80.3 & 17.0 & 2.7 & ls & 10YR 4/5 & 1.56 \\
\hline 2Btg & $48-75$ & 3.1 & 68.5 & 19.9 & 11.6 & $\mathrm{sl}$ & 10YR $4 / 4$ & 1.68 \\
\hline $2 \mathrm{Cg}$ & $75-150$ & 6.5 & 64.9 & 19.9 & 15.1 & sl & 10YR 4/6 & 1.71 \\
\hline \multicolumn{9}{|c|}{ Profile 2 - Dystric Brunic Arenosol } \\
\hline A & $0-20$ & 7.2 & 86.3 & 12.8 & 0.9 & $\mathrm{~s}$ & 7.5YR 3.5/4 & 1.40 \\
\hline Bw & $20-54$ & 10.0 & 85.0 & 13.8 & 1.1 & ls & $7.5 Y R 4 / 4$ & 1.54 \\
\hline C & $54-111$ & 13.1 & 84.0 & 9.8 & 6.1 & ls & $7.5 Y R 4 / 5$ & 1.65 \\
\hline $2 \mathrm{Cg}$ & 111-150 & 11.3 & 78.3 & 11.0 & 10.7 & $\mathrm{sl}$ & 10YR 5/3.5 & 1.82 \\
\hline \multicolumn{9}{|c|}{ Profile 3 - Dystric Brunic Arenosol } \\
\hline AEs & $0-8$ & 2.9 & 91.2 & 6.8 & 1.9 & $\mathrm{~s}$ & 10YR 3/1 & 1.35 \\
\hline Bwhs & $8-39$ & 2.2 & 91.5 & 6.7 & 1.8 & $\mathrm{~s}$ & 10YR 2.5/3 & 1.41 \\
\hline Bw & $39-63$ & 1.7 & 94.7 & 5.2 & 0.1 & $\mathrm{~s}$ & 10YR 4/6 & 1.36 \\
\hline $\mathrm{BwC}$ & $63-78$ & 2.2 & 93.6 & 6.4 & 0.1 & $\mathrm{~s}$ & 10YR 5.5/6 & 1.44 \\
\hline $\mathrm{C}$ & 78-150 & 1.6 & 91.1 & 8.4 & 0.5 & $\mathrm{~s}$ & 10YR 5/4 & 1.61 \\
\hline \multicolumn{9}{|c|}{ Profile 4 - Dystric Brunic Arenosol } \\
\hline AEs & $0-7$ & 3.0 & 91.2 & 5.9 & 2.9 & $\mathrm{~s}$ & $7.5 Y R 2 / 2$ & 1.21 \\
\hline Bwhs & $7-21$ & 1.7 & 97.5 & 2.4 & 0.0 & $\mathrm{~s}$ & 10YR $4 / 5$ & 1.41 \\
\hline Bw1 & $21-43$ & 1.3 & 89.6 & 8.7 & 1.7 & $\mathrm{~s}$ & 10YR 4/6 & 1.41 \\
\hline Bw2 & $43-53$ & 9.9 & 93.7 & 4.7 & 1.6 & $\mathrm{~s}$ & $7.5 Y R \quad 4 / 6$ & 1.54 \\
\hline C1 & $53-78$ & 0.0 & 95.4 & 4.2 & 0.4 & $\mathrm{~s}$ & 10YR 5/4 & 1.49 \\
\hline $\mathrm{C} 2$ & 78-150 & 0.0 & 95.1 & 4.9 & 0.0 & $\mathrm{~s}$ & 10YR 6/3 & 1.45 \\
\hline \multicolumn{9}{|c|}{ Profile 5 - Brunic Umbrisol } \\
\hline A1 & $0-7$ & 1.2 & 89.3 & 5.1 & 5.7 & $\mathrm{~s}$ & $7.5 Y R 2 / 1$ & 1.26 \\
\hline $\mathrm{A} 2$ & $7-30$ & 0.4 & 93.3 & 6.0 & 0.7 & $\mathrm{~s}$ & $7.5 Y R 3 / 3$ & 1.42 \\
\hline $2 \mathrm{~A}$ & 30-37 & 0.5 & 93.0 & 5.8 & 1.2 & $\mathrm{~s}$ & $7.5 Y R 3 / 2$ & 1.40 \\
\hline 2Bw & $37-60$ & 1.0 & 96.9 & 3.0 & 0.0 & $\mathrm{~s}$ & $7.5 Y R 4 / 6$ & 1.37 \\
\hline $2 \mathrm{BwC}$ & $60-77$ & 1.5 & 99.2 & 0.8 & 0.0 & $\mathrm{~s}$ & 7.5YR 4.5/6 & 1.48 \\
\hline $2 \mathrm{C} 1$ & $77-140$ & 6.1 & 99.4 & 0.6 & 0.0 & $\mathrm{~s}$ & 10YR $5 / 4$ & 1.58 \\
\hline $2 \mathrm{C} 2$ & 140-150 & 0.3 & 93.9 & 6.1 & 0.0 & $\mathrm{~s}$ & 10YR $6 / 4$ & 1.49 \\
\hline \multicolumn{9}{|c|}{ Profile 6-Dystric Brunic Arenosol } \\
\hline A & $0-18$ & 2.4 & 95.4 & 4.0 & 0.6 & $\mathrm{~s}$ & 7.5YR 4/4 & 1.41 \\
\hline Bw1 & 18-38 & 4.6 & 94.8 & 5.2 & 0.0 & $\mathrm{~s}$ & 7.5 YR 4/5 & 1.36 \\
\hline Bw2 & $38-70$ & 15.8 & 96.5 & 3.2 & 0.2 & $\mathrm{~s}$ & 7.5YR 5/7 & 1.46 \\
\hline C1 & 70-100 & 34.3 & 93.6 & 4.5 & 1.9 & $\mathrm{~s}$ & 7.5YR 4/4 & 1.47 \\
\hline $\mathrm{C} 2$ & 100-150 & 8.5 & 91.1 & 3.2 & 5.6 & $\mathrm{~S}$ & 10YR 5/4 & 1.60 \\
\hline
\end{tabular}


Table 2, continue

\begin{tabular}{|c|c|c|c|c|c|c|c|c|}
\hline Horizon & $\begin{array}{l}\text { Depth } \\
\text { [cm] }\end{array}$ & $\begin{array}{l}\text { Gravel } \\
\%\end{array}$ & $\begin{array}{l}\text { Sand } \\
\%\end{array}$ & $\begin{array}{l}\text { Silt } \\
\%\end{array}$ & $\begin{array}{l}\text { Clay } \\
\%\end{array}$ & Texture* & $\begin{array}{l}\text { Soil } \\
\text { colour }\end{array}$ & $\begin{array}{l}\text { Bulk } \\
\text { density } \\
\mathrm{g} \mathrm{cm}^{-3}\end{array}$ \\
\hline \multicolumn{9}{|c|}{ Profile 7 - Dystric Arenosol (Colluvic) } \\
\hline A1 & $0-8$ & 2.0 & 91.0 & 5.1 & 3.8 & $\mathrm{~s}$ & 10YR 2/2 & 1.26 \\
\hline $\mathrm{A} 2$ & $8-28$ & 5.6 & 95.9 & 4.1 & 0.0 & $\mathrm{~s}$ & 10YR $4 / 4$ & 1.61 \\
\hline Bw1 & $28-38$ & 2.7 & 96.3 & 3.6 & 0.1 & $\mathrm{~s}$ & 7.5 YR 4/4 & 1.48 \\
\hline A3 & $38-56$ & 2.2 & 92.9 & 6.5 & 0.6 & $\mathrm{~s}$ & 10YR 3/3.5 & 1.40 \\
\hline Bw2 & $56-63$ & 4.3 & 98.5 & 1.4 & 0.1 & $\mathrm{~s}$ & 7.5YR 4/4 & 1.39 \\
\hline $2 \mathrm{C}$ & $63-150$ & 5.5 & 99.7 & 0.2 & 0.1 & $\mathrm{~s}$ & 10YR 5/4 & 1.51 \\
\hline \multicolumn{9}{|c|}{ Profile 8 - Brunic Umbrisol (Colluvic) } \\
\hline A & $0-30$ & 2.0 & 91.3 & 7.1 & 1.6 & $\mathrm{~s}$ & 10YR 3/3 & 1.33 \\
\hline Bw & $30-46$ & 2.0 & 94.7 & 5.0 & 0.3 & $\mathrm{~s}$ & 10YR 3/4 & 1.41 \\
\hline BwC & $46-70$ & 3.4 & 95.5 & 4.4 & 0.1 & $\mathrm{~s}$ & 10YR 4/3 & 1.54 \\
\hline $2 \mathrm{Ab}$ & 70-97 & 3.5 & 96.1 & 3.9 & 0.0 & $\mathrm{~s}$ & 10YR 4/3 & 1.48 \\
\hline $2 \mathrm{~A} / \mathrm{Cb}$ & $97-120$ & 4.3 & 98.5 & 1.5 & 0.0 & $\mathrm{~s}$ & $2.5 Y 5 / 4$ & 1.47 \\
\hline $2 \mathrm{Cb}$ & $120-150$ & 3.5 & 98.3 & 1.8 & 0.0 & $\mathrm{~s}$ & $2.5 Y 5 / 4$ & 1.55 \\
\hline \multicolumn{9}{|c|}{ Profile 9 - Dystric Brunic Arenosol } \\
\hline$A(p)$ & $0-14$ & 0.5 & 93.8 & 5.5 & 0.7 & $\mathrm{~s}$ & 10YR 3/3 & 1.44 \\
\hline $\mathrm{ABw}$ & $14-31$ & 0.0 & 95.1 & 4.7 & 0.2 & $\mathrm{~s}$ & 10YR $4 / 4$ & 1.41 \\
\hline Bw & $31-58$ & 0.2 & 98.8 & 1.2 & 0.0 & $\mathrm{~s}$ & $7.5 Y R \quad 4 / 4$ & 1.64 \\
\hline $\mathrm{C} 1$ & 58-90 & 0.9 & 99.1 & 0.9 & 0.0 & $\mathrm{~s}$ & 10YR 4/6 & 1.59 \\
\hline $\mathrm{C} 2$ & $90-150$ & 0.0 & 98.8 & 1.1 & 0.0 & $\mathrm{~s}$ & 10YR 5/4 & 1.54 \\
\hline \multicolumn{9}{|c|}{ Profile 10 - Dystric Albic Brunic Arenosol } \\
\hline AEs & $0-3$ & 0.0 & 98.2 & 1.8 & 0.0 & $\mathrm{~s}$ & 10YR 4/1 & 1.48 \\
\hline $\operatorname{AEs}(p)$ & $3-22$ & 0.2 & 95.0 & 4.4 & 0.6 & $\mathrm{~s}$ & 10YR 3/4 & 1.48 \\
\hline Bwhs & $22-44$ & 0.0 & 95.6 & 4.4 & 0.0 & $\mathrm{~s}$ & 10YR $4 / 4$ & 1.37 \\
\hline $\mathrm{C} 1$ & $44-70$ & 1.0 & 98.3 & 1.7 & 0.0 & $\mathrm{~s}$ & 10YR 5/4 & 1.45 \\
\hline $\mathrm{C} 2$ & $70-150$ & 2.0 & 99.5 & 0.4 & 0.0 & $\mathrm{~s}$ & 10YR 5/4 & 1.54 \\
\hline
\end{tabular}

* s - sand; sl - sandy loam; ls - loamy sand

discontinuities. The bulk density ranged from 0.88 to $1.82 \mathrm{~g} \mathrm{~cm}^{-3}$, showing a general increasing tendency with depth.

The soils were sandy in texture, except for the 2Btg and $2 \mathrm{Cg}$ horizons in profile 1 and the $2 \mathrm{Cg}$ horizon in profile 2 , which were classed as sandy loam (Table 2). The clay content varied from 0.0 to $15.1 \%$.

\subsection{Chemical composition of the soils}

The TOC content in the $\mathrm{O}$ horizons ranged from 212.3 to $572.0 \mathrm{~g} \mathrm{~kg}^{-1}$, being the highest in the Oi horizons and the lowest in horizons Oe, or Oa. The A horizons contained 1.3-40.1 $\mathrm{g} \mathrm{kg}^{-1}$ of TOC, whereas the B horizons had 1.3-12.6 $\mathrm{g} \mathrm{kg}^{-1}$ (Table 3). The $\mathrm{N}$ content varied from 7.8 to $17.4 \mathrm{~g} \mathrm{~kg}^{-1}$ in the $\mathrm{O}$ horizons, from 0.13 to $2.13 \mathrm{~g} \mathrm{~kg}^{-1}$ in the A horizons and from 0.08 to $0.56 \mathrm{~g} \mathrm{~kg}^{-1}$ in the $\mathrm{B}$ horizons. The content of both elements generally decreased with depth. The C:N ratio varied from 22.4 to 60.0 in the ectohumus, reflecting the tree species composition of the stand and/or the site conditions. In the mineral horizons, the ratio varied from 7.0 to 22.6. The P content was from $0.55-1.54 \mathrm{~g} \mathrm{~kg}^{-1}$ in O horizons, $0.19-0.52 \mathrm{~g} \mathrm{~kg}^{-1}$ in the A horizons, $0.20-0.75 \mathrm{~g} \mathrm{~kg}^{-1}$ in the B horizons and $0.14-0.41 \mathrm{~g} \mathrm{~kg}^{-1}$ in the $\mathrm{C}$ horizons (Table 3 ). This element showed a general decreasing tendency with depth in most of the profiles.

Potassium occurred in amounts of $1.00-5.45 \mathrm{~g} \mathrm{~kg}^{-1}$ in the O horizons, 6.37-9.24 $\mathrm{g} \mathrm{kg}^{-1}$ in the A horizons, 7.09-11.65 $\mathrm{g} \mathrm{kg}^{-1}$ in the B horizons and 7.21-12.72 $\mathrm{g} \mathrm{kg}^{-1}$ in the C-horizons, showing a general increasing tendency with depth (Table 3). The richer horizons were those developed from glacial till or residual materials as compared to fluvioglacial sand or colluvial deposits. The Ca content ranged from 1.31 to $3.84 \mathrm{~g} \mathrm{~kg}^{-1}$ in the $\mathrm{O}$ horizons, from 0.66 to $1.96 \mathrm{~g} \mathrm{~kg}^{-1}$ in the solum and from 0.84 to $2.27 \mathrm{~g} \mathrm{~kg}^{-1}$ in the parent materials. Similarly, the $\mathrm{Mg}$ content was $0.44-1.21 \mathrm{~g} \mathrm{~kg}^{-1}$; 0.16-1.18 $\mathrm{g} \mathrm{kg}^{-1}$ and $0.20-1.61 \mathrm{~g} \mathrm{~kg}^{-1}$, respectively.

The $\mathrm{Ti}$ and $\mathrm{Zr}$ contents were determined and the Ti:Zr ratio was used as indicator of lithological heterogeneity. The O horizons contained 22.9-989.0 $\mathrm{mg} \mathrm{kg}^{-1}$ of Ti, whereas the mineral horizons, including the solum and parent materials, had 346.9-2521.2 $\mathrm{mg} \mathrm{kg}^{-1}$. This element was characterised by variable depth tendencies. Generally comparable tendencies were observed for $\mathrm{Zr}$, although that element occurred at several 
Table 3

$\mathrm{pH}$ and total content of organic carbon and major nutrients in the studied soils

\begin{tabular}{|c|c|c|c|c|c|c|c|c|c|c|}
\hline Horizon & $\begin{array}{l}\text { Depth } \\
{[\mathrm{cm}]}\end{array}$ & $\mathrm{pH}-\mathrm{H}_{2} \mathrm{O}$ & $\mathrm{pH}-\mathrm{KCl}$ & $\begin{array}{l}\text { TOC } \\
\mathrm{g} \mathrm{kg}^{-1}\end{array}$ & $\begin{array}{l}\mathrm{N} \\
\mathrm{g} \mathrm{kg}^{-1}\end{array}$ & $\begin{array}{l}\mathrm{P} \\
\mathrm{g} \mathrm{kg}^{-1}\end{array}$ & $\begin{array}{l}\mathrm{K} \\
\mathrm{g} \mathrm{kg}^{-1}\end{array}$ & $\begin{array}{l}\mathrm{Ca} \\
\mathrm{g} \mathrm{kg}^{-1}\end{array}$ & $\begin{array}{l}\mathrm{Mg} \\
\mathrm{g} \mathrm{kg}^{-1}\end{array}$ & $\mathrm{C}: \mathrm{N}$ \\
\hline \multicolumn{11}{|c|}{ Profile 1 - Eutric Umbric Planosol } \\
\hline Oi & $4-1$ & 4.6 & 4.0 & 479.7 & 12.89 & 0.88 & 2.85 & 2.66 & 1.21 & 37.2 \\
\hline Oe & $1-0$ & 4.8 & 4.2 & 489.9 & 16.73 & 0.97 & 2.26 & 3.84 & 1.20 & 29.3 \\
\hline A & $0-10$ & 3.4 & 2.8 & 39.0 & 2.13 & 0.45 & 9.24 & 1.26 & 0.76 & 18.3 \\
\hline$A(p)$ & $10-28$ & 4.3 & 3.6 & 11.0 & 0.65 & 0.33 & 9.24 & 1.28 & 0.61 & 16.9 \\
\hline EtBw & 28-48 & 4.5 & 3.8 & 3.2 & 0.41 & 0.24 & 10.74 & 1.53 & 1.16 & 7.8 \\
\hline 2Btg & $48-75$ & 4.9 & 3.5 & 1.7 & 0.24 & 0.22 & 11.65 & 1.27 & 0.92 & 7.0 \\
\hline $2 \mathrm{Cg}$ & $75-150$ & 4.9 & 3.2 & & & 0.30 & 12.72 & 1.44 & 1.37 & \\
\hline \multicolumn{11}{|c|}{ Profile 2 - Dystric Brunic Arenosol } \\
\hline Oi & $1-0$ & 4.1 & 3.5 & 512.2 & 15.09 & 1.12 & 2.41 & 1.85 & 0.65 & 33.9 \\
\hline A & $0-20$ & 4.1 & 3.5 & 11.7 & 0.67 & 0.27 & 8.12 & 1.57 & 0.61 & 17.6 \\
\hline Bw & $20-54$ & 4.7 & 3.8 & 4.7 & 0.36 & 0.22 & 9.39 & 1.96 & 0.91 & 13.1 \\
\hline $\mathrm{C}$ & 54-111 & 5.1 & 3.1 & & & 0.22 & 9.68 & 1.56 & 1.61 & \\
\hline $2 \mathrm{Cg}$ & 111-150 & 5.6 & 3.2 & & & 0.24 & 9.96 & 1.40 & 1.04 & \\
\hline \multicolumn{11}{|c|}{ Profile 3 - Dystric Brunic Arenosol } \\
\hline Oi & $12-7$ & 4.5 & 3.7 & 572.0 & 10.71 & 0.61 & 1.00 & 1.54 & 0.55 & 53.4 \\
\hline Oe & $7-0$ & 3.3 & 2.3 & 438.4 & 13.43 & 0.57 & 2.59 & 2.65 & 0.54 & 32.6 \\
\hline AEs & $0-8$ & 3.5 & 2.7 & 14.8 & 0.78 & 0.19 & 7.00 & 0.95 & 0.40 & 19.0 \\
\hline Bwhs & $8-39$ & 3.8 & 3.1 & 12.6 & 0.56 & 0.20 & 7.49 & 0.96 & 0.43 & 22.6 \\
\hline Bw & $39-63$ & 4.5 & 4.0 & 4.1 & 0.28 & 0.20 & 7.88 & 1.07 & 0.50 & 14.8 \\
\hline BwC & $63-78$ & 4.5 & 4.1 & 3.3 & 0.18 & 0.21 & 9.34 & 1.41 & 0.97 & 18.2 \\
\hline $\mathrm{C}$ & $78-150$ & 4.5 & 4.1 & & & 0.20 & 10.37 & 1.39 & 1.09 & \\
\hline \multicolumn{11}{|c|}{ Profile 4 - Dystric Brunic Arenosol } \\
\hline Oi & $11-6$ & 4.4 & 3.7 & 518.7 & 10.67 & 0.75 & 1.66 & 2.01 & 0.63 & 48.6 \\
\hline Oe & $6-0$ & 3.5 & 2.6 & 267.4 & 7.87 & 0.60 & 5.45 & 1.50 & 0.61 & 34.0 \\
\hline AEs & $0-7$ & 3.9 & 3.1 & 23.8 & 1.48 & 0.28 & 8.21 & 1.22 & 0.37 & 16.2 \\
\hline Bwhs & $7-21$ & 4.5 & 3.8 & 7.0 & 0.53 & 0.24 & 9.02 & 1.20 & 0.51 & 13.2 \\
\hline Bw1 & $21-43$ & 4.6 & 3.9 & 3.0 & 0.27 & 0.21 & 10.37 & 1.33 & 1.18 & 11.2 \\
\hline Bw2 & $43-53$ & 4.7 & 4.1 & 1.4 & 0.16 & 0.24 & 10.08 & 1.48 & 0.74 & 8.6 \\
\hline $\mathrm{C} 1$ & $53-78$ & 5.4 & 4.1 & & & 0.21 & 10.45 & 1.45 & 1.03 & \\
\hline $\mathrm{C} 2$ & 78-150 & 5.0 & 4.0 & & & 0.14 & 12.03 & 0.84 & 0.75 & \\
\hline \multicolumn{11}{|c|}{ Profile 5 - Brunic Umbrisol } \\
\hline Oi & $5-3$ & 4.3 & 3.6 & 536.4 & 8.94 & 0.74 & 1.28 & 1.35 & 0.49 & 60.0 \\
\hline Oe & $3-0$ & 4.4 & 3.6 & 483.2 & 12.06 & 0.93 & 2.16 & 2.30 & 0.69 & 40.1 \\
\hline A1 & $0-7$ & 3.7 & 2.9 & 40.1 & 1.91 & 0.34 & 7.70 & 1.26 & 0.34 & 20.9 \\
\hline $\mathrm{A} 2$ & $7-30$ & 4.5 & 3.9 & 9.1 & 0.49 & 0.38 & 8.08 & 1.24 & 0.59 & 18.5 \\
\hline $2 \mathrm{~A}$ & $30-37$ & 4.7 & 4.1 & 10.6 & 0.55 & 0.42 & 7.57 & 1.15 & 0.40 & 19.2 \\
\hline $2 \mathrm{Bw}$ & $37-60$ & 4.8 & 4.4 & 3.0 & 0.20 & 0.34 & 8.24 & 1.28 & 0.46 & 14.9 \\
\hline 2BwC & $60-77$ & 4.8 & 4.5 & 1.3 & 0.08 & 0.24 & 7.95 & 1.18 & 0.52 & 16.6 \\
\hline $2 \mathrm{C} 1$ & $77-140$ & 4.9 & 4.6 & & & 0.21 & 8.51 & 1.65 & 0.50 & \\
\hline $2 \mathrm{C} 2$ & 140-150 & 5.8 & 4.4 & & & 0.17 & 9.10 & 1.29 & 0.61 & \\
\hline \multicolumn{11}{|c|}{ Profile 6 - Dystric Brunic Arenosol } \\
\hline Oi & $6-4$ & 4.2 & 3.5 & 551.0 & 10.32 & 0.82 & 1.32 & 1.57 & 0.55 & 53.4 \\
\hline Oe & $4-0$ & 4.5 & 3.9 & 531.4 & 15.66 & 1.10 & 1.41 & 2.65 & 0.79 & 33.9 \\
\hline A & 0-18 & 4.4 & 3.9 & 7.6 & 0.48 & 0.24 & 7.05 & 1.15 & 0.34 & 15.8 \\
\hline Bw1 & $18-38$ & 5.0 & 4.2 & 7.1 & 0.49 & 0.32 & 7.09 & 1.27 & 0.32 & 14.4 \\
\hline Bw2 & $38-70$ & 5.4 & 4.3 & 1.5 & 0.15 & 0.28 & 7.95 & 1.60 & 0.84 & 9.7 \\
\hline C1 & 70-100 & 5.1 & 3.9 & & & 0.25 & 8.80 & 1.69 & 1.23 & \\
\hline $\mathrm{C} 2$ & 100-150 & 7.2 & 5.6 & & & 0.29 & 9.92 & 2.27 & 1.34 & \\
\hline
\end{tabular}


Table 3, continue

\begin{tabular}{|c|c|c|c|c|c|c|c|c|c|c|}
\hline Horizon & $\begin{array}{l}\text { Depth } \\
\text { [cm] }\end{array}$ & $\mathrm{pH}-\mathrm{H}_{2} \mathrm{O}$ & $\mathrm{pH}-\mathrm{KCl}$ & $\begin{array}{l}\text { TOC } \\
\text { g kg-1 }^{-1}\end{array}$ & $\begin{array}{l}\mathrm{N} \\
\mathrm{g} \mathrm{kg}^{-1}\end{array}$ & $\begin{array}{l}\mathrm{P} \\
\mathrm{g} \mathrm{kg}^{-1}\end{array}$ & $\begin{array}{l}\mathrm{K} \\
\mathrm{g} \mathrm{kg}^{-1}\end{array}$ & $\begin{array}{l}\mathrm{Ca} \\
\mathrm{g} \mathrm{kg}^{-1}\end{array}$ & $\begin{array}{l}\mathrm{Mg} \\
\mathrm{g} \mathrm{kg}^{-1}\end{array}$ & $\mathrm{C}: \mathrm{N}$ \\
\hline \multicolumn{11}{|c|}{ Profile 7 - Dystric Arenosol (Colluvic) } \\
\hline A1 & $0-8$ & 4.5 & 3.6 & 28.9 & 1.75 & 0.46 & 7.52 & 1.31 & 0.27 & 16.5 \\
\hline $\mathrm{A} 2$ & $8-28$ & 4.8 & 4.1 & 5.7 & 0.48 & 0.49 & 8.24 & 1.19 & 0.39 & 11.9 \\
\hline Bw1 & $28-38$ & 4.9 & 4.1 & 5.6 & 0.41 & 0.61 & 7.90 & 1.06 & 0.20 & 13.5 \\
\hline A3 & $38-56$ & 4.5 & 3.8 & 6.9 & 0.48 & 0.37 & 7.98 & 0.96 & 0.16 & 14.5 \\
\hline Bw2 & $56-63$ & 5.0 & 4.3 & 3.0 & 0.33 & 0.53 & 7.68 & 1.13 & 0.20 & 9.1 \\
\hline $2 \mathrm{C}$ & $63-150$ & 5.0 & 4.4 & & & 0.28 & 8.01 & 1.28 & 0.44 & \\
\hline \multicolumn{11}{|c|}{ Profile 8 - Brunic Umbrisol (Colluvic) } \\
\hline Oi & $3-0$ & 4.7 & 4.1 & 543.0 & 17.38 & 1.54 & 2.15 & 2.34 & 0.78 & 31.2 \\
\hline A & $0-30$ & 4.3 & 3.8 & 9.4 & 0.75 & 0.35 & 8.30 & 1.13 & 0.45 & 12.7 \\
\hline Bw & $30-46$ & 4.5 & 3.8 & 5.7 & 0.43 & 0.45 & 7.47 & 1.00 & 0.32 & 13.5 \\
\hline BwC & $46-70$ & 4.7 & 4.0 & 2.4 & 0.32 & 0.37 & 7.77 & 1.10 & 0.33 & 7.3 \\
\hline $2 \mathrm{Ab}$ & 70-97 & 4.8 & 3.9 & 1.9 & 0.23 & 0.25 & 7.70 & 1.09 & 0.36 & 8.1 \\
\hline $2 \mathrm{~A} / \mathrm{Cb}$ & $97-120$ & 5.1 & 4.1 & 1.3 & 0.13 & 0.26 & 8.41 & 1.38 & 0.69 & 10.2 \\
\hline $2 \mathrm{Cb}$ & $120-150$ & 5.1 & 4.2 & & & 0.19 & 8.68 & 1.50 & 0.76 & \\
\hline \multicolumn{11}{|c|}{ Profile 9 - Dystric Brunic Arenosol } \\
\hline $\mathrm{Oi}$ & $6-4$ & 4.6 & 4.2 & 456.1 & 10.92 & 1.14 & 3.55 & 1.31 & 0.56 & 41.8 \\
\hline $\mathrm{Oe}$ & $4-2$ & 4.0 & 3.0 & 248.3 & 11.10 & 0.74 & 4.40 & 1.92 & 0.52 & 22.4 \\
\hline $\mathrm{Oa}$ & $2-0$ & 3.7 & 2.6 & 368.3 & 13.87 & 0.82 & 2.83 & 1.61 & 0.48 & 26.5 \\
\hline$A(p)$ & $0-14$ & 4.7 & 4.0 & 8.5 & 0.45 & 0.47 & 7.50 & 1.08 & 0.44 & 19.0 \\
\hline $\mathrm{ABw}$ & $14-31$ & 4.8 & 4.3 & 8.8 & 0.51 & 0.52 & 7.71 & 1.11 & 0.41 & 17.1 \\
\hline Bw & $31-58$ & 5.0 & 4.5 & 1.6 & 0.13 & 0.31 & 8.33 & 1.29 & 0.43 & 12.3 \\
\hline $\mathrm{C} 1$ & 58-90 & 5.0 & 4.7 & & & 0.18 & 7.21 & 1.13 & 0.20 & \\
\hline $\mathrm{C} 2$ & $90-150$ & 5.0 & 4.7 & & & 0.15 & 7.42 & 1.15 & 0.27 & \\
\hline \multicolumn{11}{|c|}{ Profile 10 - Dystric Albic Brunic Arenosol } \\
\hline $\mathrm{Oi}$ & $6-4$ & 4.4 & 3.8 & 479.8 & 10.75 & 1.09 & 1.94 & 1.42 & 0.48 & 44.6 \\
\hline Oe & $4-2$ & 4.2 & 3.0 & 360.2 & 13.01 & 0.88 & 2.15 & 2.81 & 0.62 & 27.7 \\
\hline $\mathrm{Oa}$ & $2-0$ & 3.8 & 2.7 & 212.3 & 7.84 & 0.55 & 3.60 & 1.38 & 0.44 & 27.1 \\
\hline AEs & $0-3$ & 4.2 & 3.2 & 15.7 & 0.81 & 0.20 & 6.37 & 0.66 & 0.27 & 19.3 \\
\hline AEs(p) & $3-22$ & 4.5 & 3.7 & 5.1 & 0.29 & 0.34 & 6.66 & 0.74 & 0.18 & 17.6 \\
\hline Bwhs & $22-44$ & 4.9 & 4.4 & 5.5 & 0.37 & 0.75 & 7.10 & 1.01 & 0.30 & 14.6 \\
\hline $\mathrm{C} 1$ & $44-70$ & 5.2 & 4.4 & & & 0.41 & 8.07 & 1.54 & 0.49 & \\
\hline $\mathrm{C} 2$ & $70-150$ & 5.5 & 4.5 & & & 0.27 & 7.69 & 1.55 & 0.58 & \\
\hline
\end{tabular}

times lower concentrations the $\mathrm{Ti}$, at $0.7-99.9 \mathrm{mg} \mathrm{kg}^{-1}$ in the $\mathrm{O}$ horizons, $40.4-230.7 \mathrm{mg} \mathrm{kg}^{-1}$ in the solum and 25.2-323.9 $\mathrm{mg} \mathrm{kg}^{-1}$ in the $\mathrm{C}$ horizons.

\subsection{Forms of iron and aluminium}

The total Fe content $\left(\mathrm{Fe}_{\mathrm{t}}\right)$ varied from 0.21 to $4.95 \mathrm{~g} \mathrm{~kg}^{-1}$ in the $\mathrm{O}$ horizons and from 2.26 to $19.54 \mathrm{~g} \mathrm{~kg}^{-1}$ in the mineral horizons, including the parent materials. The richest were those horizons developed from glacial till (12.56-19.54 $\mathrm{g} \mathrm{kg}^{-1}$ ), whereas the poorest horizons were of colluvial origin (2.68-5.88 $\left.\mathrm{g} \mathrm{kg}^{-1}\right)$. The vertical distribution of the element varied among the studied profiles (Table 4). Free Fe $\left(\mathrm{Fe}_{\mathrm{d}}\right)$ occurred in amounts of 0.30 $5.96 \mathrm{~g} \mathrm{~kg}^{-1}$. Its content usually decreased with depth, except in profiles 1 and 6 . The $\mathrm{Fe}_{0}$ content ranged from 0.10 to $1.03 \mathrm{~g} \mathrm{~kg}^{-1}$, showing a general decreasing tendency with depth. The $\mathrm{Fe}_{\mathrm{d}} / \mathrm{Fe}_{\mathrm{t}}$ ratio varied from 0.04 to 0.58 , indicating variable rates of weathering of the mineral substrates. The wide spectrum of $\mathrm{Fe}_{0} / \mathrm{Fe}_{d}$ ratio indicated strongly variable degree of crystallisation of free iron oxides. This generally increased with depth.

The total $\mathrm{Al}\left(\mathrm{Al}_{\mathrm{t}}\right)$ content was higher than the $\mathrm{Fe}_{\mathrm{t}}$ content. The $O$ horizons contained $0.37-9.79 \mathrm{~g} \mathrm{~kg}^{-1}$ of that element, whereas the mineral horizons had 10.37-23.09 $\mathrm{g} \mathrm{kg}^{-1}$ (Table 4). Based on the mean values, differences between the soil horizons developed from various substrates were not great. Amorphous $\mathrm{Al}\left(\mathrm{Al}_{\mathrm{o}}\right.$ ) occurred in amounts of $0.24-3.07 \mathrm{~g} \mathrm{~kg}^{-1}$, with the highest values being in the $\mathrm{A}$ or $\mathrm{Bw}$ horizons and the lowest in the parent materials. Typically, higher $\mathrm{Al}_{0}$ contents occured in lower locations compared to the plateau or upper slopes. The $\mathrm{Al}_{\mathrm{o}} / \mathrm{Al}_{\mathrm{t}}$ ratio ranged from 0.01 to 0.22 , except the $\mathrm{O}$ horizons. 
Table 4

Iron and aluminum forms in the studied soils

\begin{tabular}{|c|c|c|c|c|c|c|c|c|c|}
\hline Horizon & $\begin{array}{l}\text { Depth } \\
\text { [cm] }\end{array}$ & $\begin{array}{l}\mathrm{Fe}_{\mathrm{t}} \\
\mathrm{g} \mathrm{kg}^{-1}\end{array}$ & $\begin{array}{l}\mathrm{Fe}_{\mathrm{d}} \\
\mathrm{g} \mathrm{kg}^{-1}\end{array}$ & $\begin{array}{l}\mathrm{Fe}_{\mathrm{o}} \\
\mathrm{g} \mathrm{kg}^{-1}\end{array}$ & $\mathrm{Fe}_{\mathrm{d}} / \mathrm{Fe}_{\mathrm{t}}$ & $\mathrm{Fe}_{\mathrm{o}} / \mathrm{Fe}_{\mathrm{d}}$ & $\begin{array}{l}\mathrm{Al}_{\mathrm{t}} \\
\mathrm{g} \mathrm{kg}^{-1}\end{array}$ & $\begin{array}{l}\mathrm{Al}_{\mathrm{o}} \\
\mathrm{g} \mathrm{kg}^{-1}\end{array}$ & $\mathrm{Al}_{\mathrm{o}} / \mathrm{Al}_{\mathrm{t}}$ \\
\hline \multicolumn{10}{|c|}{ Profile 1 - Eutric Umbric Planosol } \\
\hline Oi & $4-1$ & 1.72 & & & & & 2.35 & & \\
\hline $\mathrm{Oe}$ & $1-0$ & 1.65 & & & & & 2.34 & & \\
\hline A & $0-10$ & 7.52 & 3.49 & 1.92 & 0.46 & 0.55 & 17.79 & 1.05 & 0.06 \\
\hline$A(p)$ & $10-28$ & 8.29 & 3.71 & 1.93 & 0.45 & 0.52 & 17.60 & 1.41 & 0.08 \\
\hline EtBw & $28-48$ & 10.12 & 3.52 & 1.58 & 0.35 & 0.45 & 23.09 & 1.18 & 0.05 \\
\hline $2 \mathrm{Btg}$ & $48-75$ & 16.12 & 4.84 & 1.29 & 0.30 & 0.27 & 18.89 & 0.65 & 0.03 \\
\hline $2 \mathrm{Cg}$ & $75-150$ & 19.54 & 5.96 & 1.11 & 0.30 & 0.19 & 20.77 & 0.57 & 0.03 \\
\hline \multicolumn{10}{|c|}{ Profile 2 - Dystric Brunic Arenosol } \\
\hline Oi & $1-0$ & 0.30 & & & & & 0.45 & & \\
\hline A & $0-20$ & 7.93 & 4.20 & 1.47 & 0.53 & 0.35 & 16.21 & 1.17 & 0.07 \\
\hline Bw & $20-54$ & 9.84 & 3.50 & 0.55 & 0.36 & 0.16 & 13.52 & 0.87 & 0.06 \\
\hline $\mathrm{C}$ & $54-111$ & 10.17 & 2.86 & 0.78 & 0.28 & 0.27 & 21.63 & 0.45 & 0.02 \\
\hline $2 \mathrm{Cg}$ & $111-150$ & 12.56 & 0.47 & 0.43 & 0.04 & 0.91 & 13.89 & 0.44 & 0.03 \\
\hline \multicolumn{10}{|c|}{ Profile 3 - Dystric Brunic Arenosol } \\
\hline Oi & $12-7$ & 0.21 & & & & & 0.37 & & \\
\hline $\mathrm{Oe}$ & $7-0$ & 3.11 & & & & & 6.32 & & \\
\hline AEs & $0-8$ & 4.64 & 2.55 & 1.50 & 0.55 & 0.59 & 12.45 & 0.52 & 0.04 \\
\hline Bwhs & $8-39$ & 4.90 & 2.76 & 1.89 & 0.56 & 0.68 & 13.63 & 0.92 & 0.07 \\
\hline Bw & $39-63$ & 5.14 & 2.47 & 1.35 & 0.48 & 0.55 & 15.32 & 1.73 & 0.11 \\
\hline $\mathrm{BwC}$ & $63-78$ & 6.33 & 2.12 & 1.04 & 0.34 & 0.49 & 18.27 & 1.66 & 0.09 \\
\hline $\mathrm{C}$ & 78-150 & 6.56 & 1.76 & 0.65 & 0.27 & 0.37 & 18.35 & 0.92 & 0.05 \\
\hline \multicolumn{10}{|c|}{ Profile 4 - Dystric Brunic Arenosol } \\
\hline Oi & $11-6$ & 0.85 & & & & & 1.24 & & \\
\hline Oe & $6-0$ & 4.95 & & & & & 9.79 & & \\
\hline AEs & $0-7$ & 5.80 & 2.34 & 1.18 & 0.40 & 0.50 & 14.03 & 0.77 & 0.05 \\
\hline Bwhs & $7-21$ & 7.37 & 2.45 & 1.10 & 0.33 & 0.45 & 14.58 & 1.52 & 0.10 \\
\hline Bw1 & $21-43$ & 8.14 & 2.28 & 0.63 & 0.28 & 0.28 & 19.51 & 1.11 & 0.06 \\
\hline Bw2 & $43-53$ & 9.02 & 2.88 & 0.53 & 0.32 & 0.19 & 17.15 & 1.00 & 0.06 \\
\hline $\mathrm{C} 1$ & $53-78$ & 7.94 & 1.79 & 0.54 & 0.23 & 0.30 & 15.04 & 0.64 & 0.04 \\
\hline $\mathrm{C} 2$ & $78-150$ & 5.37 & 0.68 & 0.22 & 0.13 & 0.32 & 15.04 & 0.35 & 0.02 \\
\hline \multicolumn{10}{|c|}{ Profile 5 - Brunic Umbrisol } \\
\hline Oi & $5-3$ & 0.27 & & & & & 0.55 & & \\
\hline Oe & $3-0$ & 1.57 & & & & & 2.17 & & \\
\hline $\mathrm{A} 1$ & $0-7$ & 4.97 & 2.66 & 1.35 & 0.54 & 0.51 & 13.79 & 0.77 & 0.06 \\
\hline A2 & $7-30$ & 5.44 & 2.83 & 1.36 & 0.52 & 0.48 & 15.38 & 1.32 & 0.09 \\
\hline $2 \mathrm{~A}$ & $30-37$ & 4.91 & 2.84 & 1.39 & 0.58 & 0.49 & 15.14 & 1.81 & 0.12 \\
\hline $2 \mathrm{Bw}$ & $37-60$ & 4.54 & 2.11 & 0.81 & 0.46 & 0.38 & 16.14 & 2.21 & 0.14 \\
\hline 2BwC & $60-77$ & 3.44 & 1.54 & 0.47 & 0.45 & 0.30 & 14.27 & 1.18 & 0.08 \\
\hline $2 \mathrm{C} 1$ & $77-140$ & 2.99 & 0.84 & 0.30 & 0.28 & 0.35 & 15.75 & 0.50 & 0.03 \\
\hline $2 \mathrm{C} 2$ & $140-150$ & 3.56 & 0.60 & 0.25 & 0.17 & 0.42 & 14.45 & 0.51 & 0.04 \\
\hline \multicolumn{10}{|c|}{ Profile 6 - Dystric Brunic Arenosol } \\
\hline Oi & $6-4$ & 0.30 & & & & & 0.63 & & \\
\hline $\mathrm{Oe}$ & $4-0$ & 0.73 & & & & & 1.21 & & \\
\hline A & $0-18$ & 4.74 & 2.24 & 1.20 & 0.47 & 0.53 & 12.96 & 1.11 & 0.09 \\
\hline Bw1 & $18-38$ & 5.69 & 2.76 & 1.31 & 0.48 & 0.47 & 14.92 & 2.21 & 0.15 \\
\hline Bw2 & $38-70$ & 8.13 & 2.00 & 0.45 & 0.25 & 0.22 & 15.91 & 1.33 & 0.08 \\
\hline $\mathrm{C} 1$ & $70-100$ & 10.41 & 3.00 & 0.54 & 0.29 & 0.18 & 16.89 & 0.75 & 0.04 \\
\hline $\mathrm{C} 2$ & 100-150 & 8.51 & 2.18 & 0.36 & 0.26 & 0.16 & 19.27 & 0.24 & 0.01 \\
\hline
\end{tabular}


Table 4, continue

\begin{tabular}{|c|c|c|c|c|c|c|c|c|c|}
\hline Horizon & $\begin{array}{l}\text { Depth } \\
{[\mathrm{cm}]}\end{array}$ & $\begin{array}{l}\mathrm{Fe}_{\mathrm{t}} \\
\mathrm{g} \mathrm{kg}^{-1}\end{array}$ & $\begin{array}{l}\mathrm{Fe}_{\mathrm{d}} \\
\mathrm{g} \mathrm{kg}^{-1}\end{array}$ & $\begin{array}{l}\mathrm{Fe}_{\mathrm{o}} \\
\mathrm{g} \mathrm{kg}^{-1}\end{array}$ & $\mathrm{Fe}_{\mathrm{d}} / \mathrm{Fe}_{\mathrm{t}}$ & $\mathrm{Fe}_{\mathrm{o}} / \mathrm{Fe}_{\mathrm{d}}$ & $\begin{array}{l}\mathrm{Al}_{\mathrm{t}} \\
\mathrm{g} \mathrm{kg}^{-1}\end{array}$ & $\begin{array}{l}\mathrm{Al}_{\circ} \\
\mathrm{g} \mathrm{kg}^{-1}\end{array}$ & $\mathrm{Al}_{\mathrm{o}} / \mathrm{Al}_{\mathrm{t}}$ \\
\hline \multicolumn{10}{|c|}{ Profile 7 - Dystric Arenosol (Colluvic) } \\
\hline A1 & $0-8$ & 4.43 & 2.00 & 1.08 & 0.45 & 0.54 & 13.56 & 0.83 & 0.06 \\
\hline A2 & $8-28$ & 5.88 & 2.43 & 1.22 & 0.41 & 0.50 & 15.42 & 1.75 & 0.11 \\
\hline Bw1 & $28-38$ & 4.98 & 2.61 & 1.79 & 0.52 & 0.68 & 15.01 & 2.12 & 0.14 \\
\hline A3 & $38-56$ & 4.31 & 2.20 & 1.10 & 0.51 & 0.50 & 13.83 & 0.75 & 0.05 \\
\hline Bw2 & $56-63$ & 4.63 & 2.12 & 1.22 & 0.46 & 0.57 & 14.36 & 2.04 & 0.14 \\
\hline $2 \mathrm{C}$ & $63-150$ & 4.01 & 1.17 & 0.36 & 0.29 & 0.30 & 14.40 & 0.74 & 0.05 \\
\hline \multicolumn{10}{|c|}{ Profile 8 - Brunic Umbrisol (Colluvic) } \\
\hline $\mathrm{Oi}$ & $3-0$ & 0.60 & & & & & 0.99 & & \\
\hline A & $0-30$ & 5.02 & 2.52 & 1.43 & 0.50 & 0.57 & 15.27 & 0.99 & 0.07 \\
\hline Bw & $30-46$ & 3.84 & 1.85 & 1.28 & 0.48 & 0.69 & 13.30 & 1.01 & 0.08 \\
\hline BwC & $46-70$ & 2.68 & 0.83 & 0.48 & 0.31 & 0.58 & 13.80 & 0.68 & 0.05 \\
\hline $2 \mathrm{Ab}$ & $70-97$ & 2.45 & 0.42 & 0.26 & 0.17 & 0.62 & 13.47 & 0.65 & 0.05 \\
\hline $2 \mathrm{~A} / \mathrm{Cb}$ & $97-120$ & 3.40 & 0.58 & 0.25 & 0.17 & 0.42 & 15.40 & 0.92 & 0.06 \\
\hline $2 \mathrm{Cb}$ & $120-150$ & 3.39 & 0.38 & 0.10 & 0.11 & 0.28 & 15.50 & 0.67 & 0.04 \\
\hline \multicolumn{10}{|c|}{ Profile 9 - Dystric Brunic Arenosol } \\
\hline $\mathrm{Oi}$ & $6-4$ & 0.21 & & & & & 0.46 & & \\
\hline $\mathrm{Oe}$ & $4-2$ & 2.82 & & & & & 7.79 & & \\
\hline $\mathrm{Oa}$ & $2-0$ & 3.00 & & & & & 6.46 & & \\
\hline$A(p)$ & $0-14$ & 4.86 & 2.36 & 1.54 & 0.49 & 0.65 & 13.93 & 1.33 & 0.10 \\
\hline $\mathrm{ABw}$ & $14-31$ & 4.54 & 2.22 & 1.54 & 0.49 & 0.69 & 15.63 & 2.82 & 0.18 \\
\hline Bw & $31-58$ & 3.93 & 1.21 & 0.74 & 0.31 & 0.61 & 14.76 & 1.37 & 0.09 \\
\hline $\mathrm{C} 1$ & 58-90 & 2.47 & 0.80 & 0.43 & 0.32 & 0.53 & 11.81 & 0.59 & 0.05 \\
\hline $\mathrm{C} 2$ & $90-150$ & 2.26 & 0.49 & 0.23 & 0.22 & 0.47 & 12.52 & 0.52 & 0.04 \\
\hline \multicolumn{10}{|c|}{ Profile 10 - Dystric Albic Brunic Arenosol } \\
\hline $\mathrm{Oi}$ & $6-4$ & 0.31 & & & & & 0.50 & & \\
\hline $\mathrm{Oe}$ & $4-2$ & 1.89 & & & & & 3.72 & & \\
\hline $\mathrm{Oa}$ & $2-0$ & 2.67 & & & & & 6.15 & & \\
\hline AEs & $0-3$ & 2.39 & 0.85 & 0.42 & 0.35 & 0.49 & 10.37 & 0.25 & 0.02 \\
\hline $\operatorname{AEs}(p)$ & $3-22$ & 3.42 & 1.50 & 0.90 & 0.44 & 0.60 & 10.52 & 0.57 & 0.05 \\
\hline Bwhs & $22-44$ & 4.00 & 1.67 & 1.49 & 0.42 & 0.89 & 13.76 & 3.07 & 0.22 \\
\hline $\mathrm{C} 1$ & $44-70$ & 4.30 & 0.31 & 0.29 & 0.07 & 0.93 & 13.49 & 1.63 & 0.12 \\
\hline $\mathrm{C} 2$ & $70-150$ & 2.54 & 0.30 & 0.12 & 0.12 & 0.38 & 14.71 & 0.84 & 0.06 \\
\hline
\end{tabular}

\subsection{Sorptive properties and $\mathrm{pH}$}

The reaction of the soils varied from strongly acidic to close to neutral ( $\mathrm{pH}-\mathrm{H}_{2} \mathrm{O} 3.3-7.2 ; \mathrm{pH}-\mathrm{KCl}$ 2.3-5.6). The lowest $\mathrm{pH}$ was typically noted in the Oe and $\mathrm{Oa}$ ectohumus sub-horizons or the A horizons (Table 3). There the $\mathrm{pH}$ increased with depth and was the highest in the parent material. The acidic nature of the soils was confirmed by the ionic composition of the soil sorption complex. In most cases $\mathrm{H}^{+}$and $\mathrm{Al}^{3+}$ predominated over basic cations, except in the Btg and $\mathrm{Cg}$ horizons in profile 1 , the $\mathrm{Cg}$ horizon in profile 2 and the $\mathrm{C} 2$ horizon in profile 6 (Table 5). The $\mathrm{H}_{\mathrm{w}}$ varied from 0.05 to $4.77 \mathrm{cmol}_{(+)} \mathrm{kg}^{-1}$, whereas TEB ranged from 0.03 to $3.79 \mathrm{cmol}_{(+)} \mathrm{kg}^{-1}$. In all the soils, $\mathrm{Ca}^{2+}$ was the major basic cation. The CEC varied from 0.08 to $5.84 \mathrm{cmol}_{(+)}$ $\mathrm{kg}^{-1}$, and was positively correlated with clay $(\mathrm{r}=0.721)$ and TOC $(r=0.746)$. It was the highest in the A horizons, decreasing with depth. There was also a clear spatial tendency, with higher CEC values noted in the plateau soils developed from glacial till or residual materials as compared to fluvioglacial sands or colluvic materials. 
Table 5

Sorptive characteristics of the studied soils




Table 5, continue

\begin{tabular}{|c|c|c|c|c|c|c|c|c|c|}
\hline \multicolumn{10}{|c|}{ Profile 7 - Dystric Arenosol (Colluvic) } \\
\hline A1 & $0-8$ & 0.02 & 0.10 & 1.53 & 0.33 & 2.39 & 0.98 & 4.37 & 66.9 \\
\hline $\mathrm{A} 2$ & $8-28$ & 0.01 & 0.03 & 0.04 & 0.01 & 1.39 & 0.69 & 1.47 & 11.0 \\
\hline Bw1 & $28-38$ & 0.01 & 0.03 & 0.04 & 0.01 & 0.84 & 0.43 & 0.93 & 17.1 \\
\hline A3 & $38-56$ & 0.01 & 0.02 & 0.06 & 0.01 & 1.40 & 0.70 & 1.51 & 12.8 \\
\hline Bw2 & $56-63$ & 0.03 & 0.02 & 0.03 & 0.01 & 0.51 & 0.25 & 0.60 & 26.5 \\
\hline $2 \mathrm{C}$ & $63-150$ & 0.00 & 0.01 & 0.02 & 0.01 & 0.35 & 0.15 & 0.40 & 24.6 \\
\hline \multicolumn{10}{|c|}{ Profile 8 - Brunic Umbrisol (Colluvic) } \\
\hline A & $0-30$ & 0.01 & 0.03 & 0.07 & 0.02 & 1.78 & 0.86 & 1.92 & 13.9 \\
\hline Bw & $30-46$ & 0.01 & 0.01 & 0.04 & 0.01 & 1.24 & 0.58 & 1.31 & 11.3 \\
\hline BwC & $46-70$ & 0.01 & 0.01 & 0.03 & 0.01 & 0.84 & 0.39 & 0.91 & 13.4 \\
\hline $2 \mathrm{Ab}$ & 70-97 & 0.02 & 0.01 & 0.03 & 0.01 & 1.10 & 0.52 & 1.18 & 13.0 \\
\hline $2 \mathrm{~A} / \mathrm{Cb}$ & $97-120$ & 0.01 & 0.01 & 0.03 & 0.01 & 0.73 & 0.34 & 0.80 & 16.1 \\
\hline $2 \mathrm{Cb}$ & $120-150$ & 0.01 & 0.02 & 0.03 & 0.03 & 0.54 & 0.27 & 0.63 & 23.3 \\
\hline \multicolumn{10}{|c|}{ Profile 9 - Dystric Brunic Arenosol } \\
\hline$A(p)$ & 0-14 & 0.01 & 0.03 & 0.04 & 0.02 & 0.93 & 0.50 & 1.03 & 16.2 \\
\hline $\mathrm{ABw}$ & 14-31 & 0.01 & 0.01 & 0.04 & 0.01 & 0.47 & 0.25 & 0.54 & 21.5 \\
\hline Bw & $31-58$ & 0.01 & 0.01 & 0.01 & 0.01 & 0.15 & 0.09 & 0.19 & 27.3 \\
\hline $\mathrm{C} 1$ & 58-90 & 0.01 & 0.01 & 0.01 & 0.01 & 0.05 & 0.03 & 0.08 & 53.6 \\
\hline $\mathrm{C} 2$ & 90-150 & 0.01 & 0.01 & 0.01 & 0.01 & 0.10 & 0.06 & 0.13 & 37.4 \\
\hline \multicolumn{10}{|c|}{ Profile 10 - Dystric Albic Brunic Arenosol } \\
\hline AEs & $0-3$ & 0.02 & 0.05 & 0.09 & 0.05 & 1.40 & 0.56 & 1.61 & 27.1 \\
\hline $\operatorname{AEs}(p)$ & $3-22$ & 0.01 & 0.02 & 0.03 & 0.02 & 0.96 & 0.47 & 1.03 & 13.9 \\
\hline Bwhs & $22-44$ & 0.01 & 0.01 & 0.04 & 0.01 & 0.47 & 0.25 & 0.54 & 21.3 \\
\hline $\mathrm{C} 1$ & $44-70$ & 0.01 & 0.01 & 0.02 & 0.01 & 0.36 & 0.21 & 0.42 & 20.6 \\
\hline $\mathrm{C} 2$ & $70-150$ & 0.01 & 0.01 & 0.02 & 0.01 & 0.14 & 0.08 & 0.18 & 32.8 \\
\hline
\end{tabular}

*effective saturation with bases

\section{Discussion}

Parent material origin and relief are considered to be highly important factors influencing the spatio-temporal variability of soil cover at various scales (Jenny, 1941; Konecka-Betley et al., 1994; Janowska, 2001; Jonczak et al., 2013). These factors directly influence soil-forming processes and many soil characteristics. Moreover, indirect effects can impact the water regime (Moeslund et al., 2013), vegetation (Florinsky and Kuryakova, 1996) and microclimatic conditions (Macyk et al., 1978) among other things. The role of the parent material is mainly as a substrate for soil-forming processes. It has certain properties that determine, within a certain range, the direction of soil development. Meanwhile, relief is considered to be factor in internally differentiating sediments from various depositional environments and determining slope processes. The strong linkage between these two factors, and the linkage between them and the other factors involved in soil formation determine large heterogeneity in the soil cover over post-glacial landscapes. Brunic Arenosols are common components of such landscapes.

The studied profiles covered soils with a wide spectrum of morphological, physical and chemical characteristics. Eutric Umbric Planosols from the plateau (profile 1) were characterised by their bipartite texture. According to current understanding, the vertical variability of textural parameters in that soil reference group (and related groups, according to the WRB classification) can result from lessivage and/or lithological discontinuity (Świtoniak, 2006). Contrasting differences between the upper (A, $\mathrm{A}(\mathrm{p})$ and EtBw) and underlying (2Btg and $2 \mathrm{Cg}$ ) horizons, in terms of texture and a sharp transition from the EtBw into the 2Btg horizon, indicate a greater importance of lithology over lessivage in the studied case. Considering the low vertical variability in the Ti:Zr ratio (Fig. 2), it can be stated that glacial till (present in the 2Btg and 2Cg horizons) was also the primary source of 


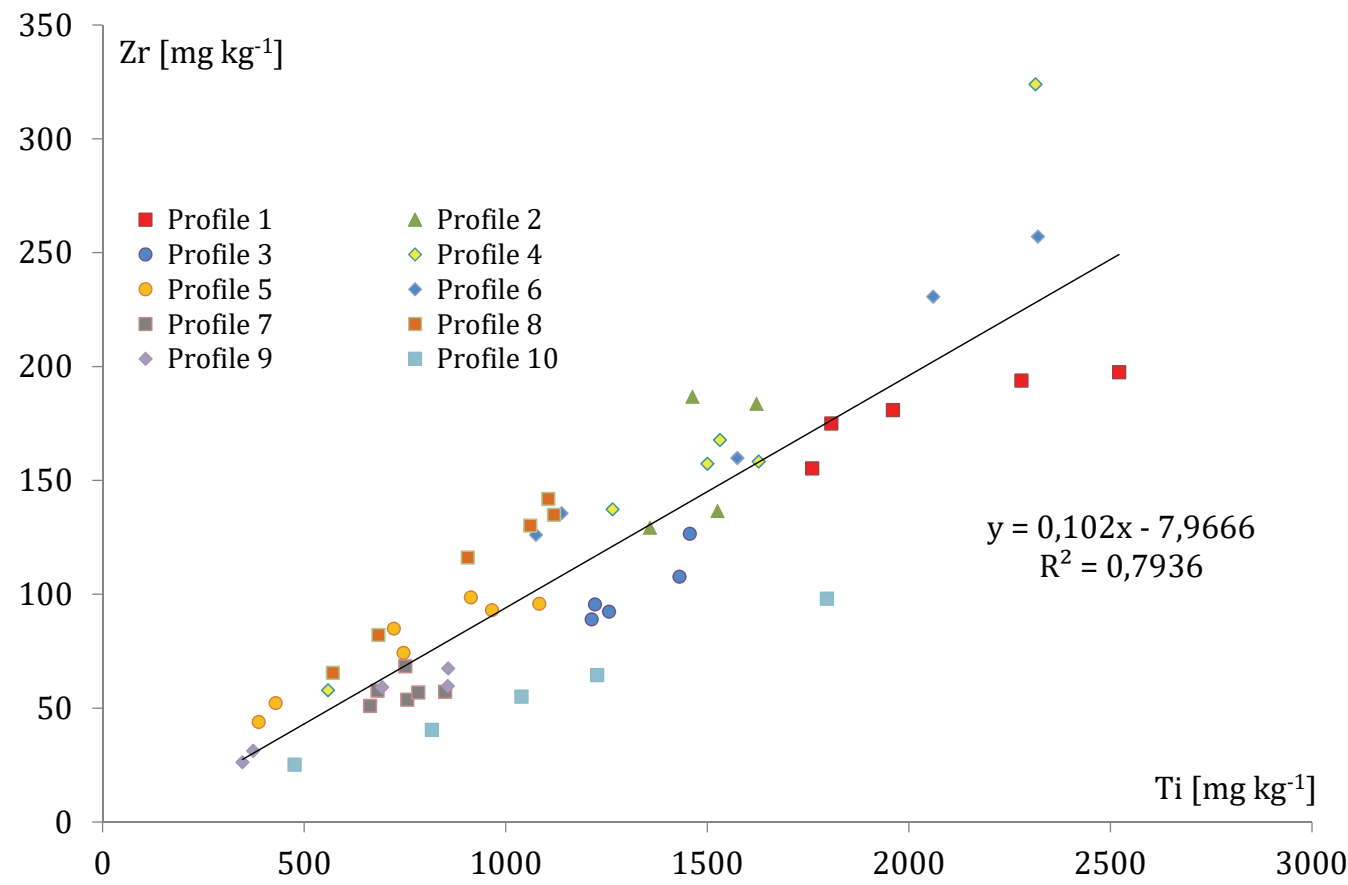

Fig. 2. Correlation between total contents of $\mathrm{Ti}$ and $\mathrm{Zr}$ in the studied soils excluding O-horizons the mineral substrates for the upper horizons. The top layer of till was transformed by fluvial processes, although redeposition of these sediments occurred only on local scale. The Ti and $\mathrm{Zr}$ contents were strongly positively correlated, and the Ti:Zr ratio was characterised by relatively low variability (7.1-14.9 in the mineral horizons of profiles 1-9). The ratio was slightly higher in profile 10 , which was located in a transitional zone between the valley slope and the supra-flood terrace. The land elevations dissected by dry valleys are covered by coarse-textured, gravel-enriched (Table 2) residual materials overlying glacial till (profile 2). Typically, Dystric Brunic Arenosols occur in such locations. These soils are also typical in slope locations. However, their morphology and certain characteristics varied along the slope, with soils from an upper location (profile 3 ) and the transitional zone between the valley slope and the supra-flood terrace (profile 10) being characterised by thinner sola compared to the lower and middle slopes or valley bottom (profiles 4, 6, 9). The presence of colluvial horizons in some profiles constituted evidence of downslope sediment transport. Deep A horizons (Umbric) in profile 8 represented an effect of that process, with the soil being classed as a Brunic Umbrisol (Colluvic). Erosion intensification probably resulted in denudation of the primary soil in profile 7 , followed by covering by stratified colluvium (Fig. 1).

The Bw horizons varied greatly in the profiles, including in terms of thickness and colour. The transition into the $\mathrm{C}$ horizon was usually gradual, which is typical for this soil reference group (Konecka-Betley et al., 1994; Degórski, 2002; Brożek and Zwydak, 2010). In profile 4, located on the slope, the transition was sharp. This might suggest relocation of only the developed Bw horizon, constituting evidence of strong erosion in the post-glacial landscape, with advanced pedogenesis. The observed spatial variability in the advancement of the soil-forming processes in the Brunic Arenosols, based on morphological features, was confirmed by the chemical indicators. The $\mathrm{Fe}_{\mathrm{t}}$ and
$\mathrm{Al}_{\mathrm{t}}$ contents were relatively low (Table 5), although typical of soils developed from sandy materials (Janowska, 2001; Brożek and Zwydak, 2010; Jonczak et al., 2013). The $\mathrm{Fe}_{\mathrm{d}}$ content varied among the profile locations and horizons, showing a general downslope decreasing tendency. The vertical distribution of $\mathrm{Fe}_{\mathrm{d}}$ in profile 1 also suggests a considerable intensity of weathering in the upper parts of the parent material. This observation requires more detailed study. In the Brunic Arenosols, the highest $\mathrm{Fe}_{\mathrm{d}}$ contents were noted in the $\mathrm{B}$ or A horizons. The $\mathrm{Fe}_{\mathrm{d}} / \mathrm{Fe}_{\mathrm{t}}$ ratio showed great vertical variability in the profiles and low spatial variability in the studied sequence. The spatial differences between the soils are more evident when comparing the stocks of $\mathrm{Fe}_{\mathrm{d}}$ in soil pedons (1 x $1 \mathrm{~m}$ wide and $1.5 \mathrm{~m}$ deep) (Table 6). A clear downslope decreasing tendency, from $12.23 \mathrm{~kg} \mathrm{~m}^{-2}$ in profile 1 to $1.45 \mathrm{~kg} \mathrm{~m}^{-2}$ in profile 10, was recorded. Malczyk (1988) reported a different distribution pattern for $\mathrm{Fe}_{\mathrm{t}}$ in an aeolian landscape, with the lowest content occurring in the dune top and the highest on the slope foot.

The $\mathrm{Fe}_{\mathrm{o}} / \mathrm{Fe}_{\mathrm{d}}$ ratio, as a measure of free iron amorphism (Konecka-Betley, 1968; Pokojska, 1979), showed great variability in the studied soils (Table 4). Typically, the highest values were recorded in the A horizons, indicating the poor crystallisation of sesquioxides, and the values then decreased with depth. This is a common tendency in Brunic Arenosols. Poor crystallisation in the A horizon can be explained as an inhibitory effect caused by humic substances (Cornell and Schwertmann, 1979). Generally, soil profiles in lower relief locations are characterised by lower sesquioxide crystallinity than in higher locations. This is probably result of a higher moisture content and the downslope transport of $\mathrm{Fe}_{\mathrm{o}}$ following its accumulation in depressions in the landscape. This process "lateral podsolization" is common in hilly landscapes, resulting in the formation of reddish horizons enriched in sesquioxides (Sommer et al., 2000; Jankowski, 2014b). Soils containing such horizons were noted in locations 
Table 6

Stocks of selected components in the studied soils up to $150 \mathrm{~cm}$ excluding O-horizons

\begin{tabular}{|c|c|c|c|c|c|c|c|c|c|c|c|}
\hline \multirow[t]{2}{*}{ Component } & \multirow[t]{2}{*}{ Unit } & \multicolumn{10}{|c|}{ Profile number } \\
\hline & & 1 & 2 & 3 & 4 & 5 & 6 & 7 & 8 & 9 & 10 \\
\hline TOC & $\mathrm{kg} \mathrm{m}^{-2}$ & 8.08 & 5.76 & 9.19 & 4.57 & 8.81 & 4.55 & 7.62 & 7.14 & 4.51 & 3.78 \\
\hline $\mathrm{N}$ & $\mathrm{kg} \mathrm{m}^{-2}$ & 0.59 & 0.38 & 0.46 & 0.34 & 0.47 & 0.33 & 0.54 & 0.65 & 0.27 & 0.23 \\
\hline $\mathrm{P}$ & $\mathrm{kg} \mathrm{m}^{-2}$ & 0.69 & 0.58 & 0.45 & 0.40 & 0.59 & 0.62 & 0.81 & 0.66 & 0.59 & 0.81 \\
\hline $\mathrm{Fe}_{\mathrm{d}}$ & $\mathrm{kg} \mathrm{m}^{-2}$ & 12.23 & 6.04 & 4.79 & 3.21 & 3.41 & 5.33 & 3.67 & 2.27 & 2.40 & 1.45 \\
\hline $\mathrm{H}_{\mathrm{w}}$ & $\mathrm{mol}_{(+)} \mathrm{m}^{-2}$ & 54.82 & 41.48 & 28.55 & 29.66 & 9.90 & 9.83 & 16.76 & 22.46 & 4.88 & 7.86 \\
\hline TEB & $\mathrm{mol}_{(+)} \mathrm{m}^{-2}$ & 59.98 & 44.54 & 2.38 & 3.50 & 2.43 & 27.32 & 3.40 & 1.86 & 1.00 & 1.21 \\
\hline CEC & $\mathrm{mol}_{(+)} \mathrm{m}^{-2}$ & 114.80 & 86.02 & 30.93 & 33.16 & 12.33 & 37.15 & 20.17 & 24.32 & 5.88 & 9.07 \\
\hline
\end{tabular}

around the studied area. The downslope transport of sesquioxides was also confirmed by the distribution of $\mathrm{Al}_{0}$, with higher concentrations being typical of lower, rather than higher, locations. The vertical transport of $\mathrm{Al}_{o}$, constituting evidence of podsolization was seen in the majority of the studied profiles. The displacement of labile $\mathrm{Al}$ fractions is an indicator of the early stages of podsolisation, before any visual effects have developed (Bednarek, 1991). Moreover, Kuźnicki and Skłodowski (1974) stated that podsolization intensity in Brunic Arenosols is strongly controlled by the origin of the parent material, whereas Konecka-Betley (1977) highlighted the importance of the presence of humic substances, particularly fulvic acids.

The accumulation of organic matter is an inherent process in soil formation. Sandy soils, including Brunic Arenosols, are usually poor in that component. The studied soils varied greatly in terms of TOC content (Table 3) and stocks (Table 6). The highest stocks were generally noted in the soils from higher locations and from the slope foot. A limited impact of water erosion on the soils from the upper locations and the deposition of organic matter rich colluvic materials at the slope foot locations are likely the major factors influencing the large accumulations of TOC in those soils. There were comparable tendencies in the spatial distributions of TOC and N, which were strongly positively correlated, particularly in the mineral horizons $(r=0.983)$. The spatial variability of the C:N ratio indicates heterogeneity in the studied environment, in terms of trophic conditions. Typically, $\mathrm{P}$ occurred in its highest amounts in the $\mathrm{O}$ horizons and in its lowest amounts in the parent material. The observed general downslope increasing tendency in the $\mathrm{P}$ contents and stocks in the mineral horizons suggests lateral transport of that element and its accumulation in depressions in the landscape. Irrespective of the observed spatial variability, the P contents in the studied soils is considered to be low, and comparable to observations by other authors on Brunic Arenosols (Okołowicz et al., 2003; Brożek and Zwydak, 2010). Sandy substrates are also usually poor in K, Ca and Mg (Kuźnicki et al., 1974), and this is confirmed here (Table 3). These elements are usually more enriched in Brunic Arenosols influenced by high groundwater levels (Kowalkowski, 1977b).

Brunic Arenosols are typically acidic soils (Janowska, 2001; Brożek and Zwydak, 2010). All the studied soils showed increasing $\mathrm{pH}$ with depth in their mineral horizons. This can be explained by the percolative water regime and the influence of forest vegetation as a source of the acidity (Augusto et al., 2002). Spatial variability was unexpectedly low in this regard. Considering the soil $\mathrm{pH}$, a predominance of acidic ions in the soil sorption complex would be expected. This was confirmed here for the majority of horizons. More than $50 \%$ of basic cations were recorded only in the parent materials of profiles 1, 2, 3, 5 and 6 . There was an interesting spatial distribution of acidic ion stocks in the soil pedons of up to $150 \mathrm{~cm}$. Much higher stocks were observed in the upper locations, followed by the mid-slopes and the lowest stocks in the foot slope locations, suggesting a significant role of relief as a factor influencing soil sorption. This tendency is clearer from the sum of the acidic and basic cations (Table 6). The importance of relief as a factor in soil sorptive properties is still poorly understood. Its role can be viewed in the context of erosion and downslope sediment transport. A high positive correlation between CEC and TOC content highlights the significant role of humic substances.

\section{Conclusions}

Our findings confirm the significant roles of lithology, relief and slope processes as factors influencing the formation and spatial variability of soil cover in young glacial landscapes. The soil cover of the studied toposequence was dominated by Dystric Brunic Arenosols, Dystric Albic Brunic Arenosols and Brunic Umbrisols. The origin of the parent material, and its transformation by water erosion and other processes typical of slopes, strongly influenced the soil morphology and various other characteristics along the studied slope. Although the spatial and vertical variability of the textural parameters suggested varied origins for the soil mineral substrates, the low variability of the Ti:Zr ratio confirmed only a local-scale transformation of soil mineral substrates. Typically, the soils from the upper slope locations and the transitional zone between the valley slope and supra-flood terrace were characterised by thinner sola compared to the soils from the slope foot, the middle slope or the valley bottom. The presence of colluvial horizons in some locations constituted evidence of downslope sediment transport. The brown or rust coloured B horizons enriched in pedogenic forms of $\mathrm{Fe}$ and $\mathrm{Al}$ constituted evidence of strong weathering. The spatial distribution 
of sesquioxides suggested their lateral transport. The soils varied greatly in terms of TOC content and stock. The highest stocks were noted in the soils from higher locations and from the slope foot, and were lowest in the mid-slope locations, highlighting the importance of erosion as a factor influencing soil organic matter accumulation. A comparable tendency was seen for $\mathrm{N}$, and both $\mathrm{N}$ and TOC were strongly positively correlated. The contents of $\mathrm{P}, \mathrm{K}, \mathrm{Ca}$ and $\mathrm{Mg}$ were low, as is typical of sandy soils developed from fluvioglacial substrates. All of the soils had $\mathrm{pH}$ values and sorptive characteristics typical of Brunic Arenosols, although their spatial variability was large. Higher CEC values were recorded in the higher locations, followed by the upper and midslopes, with the lowest found at the slope foot.

\section{References}

Andrzejczyk, T., Sewerniak, P., 2016. Gleby i siedliska drzewostanów nasiennych dębu szypułkowego (Quercus robur) i dębu bezszypułkowego (Q. petraea) w Polsce. Sylwan 160(8), 674-683.

Augusto, L., Ranger, J., Binkley, D., Rothe, A., 2002. Impact of several common tree species of European temperate forests on soil fertility. Annals of Forest Science 59(3), 233-253. https://doi.org/10.1051/forest: 2002020

Banaszuk, H., 1977. Geneza i ewolucja pokrywy glebowej na wydmach Kotliny Biebrzańskiej. Roczniki Gleboznawcze - Soil Science Annual 30(2), 111-142. (In Polish with English summary)

Bednarek, R., 1991. Wiek, geneza i stanowisko systematyczne gleb rdzawych w świetle badań paleopedologicznych w okolicach Osia. Wydawnictwo UMK, Toruń.

Biały, K., 1999. Dowolność wyróżniania typów siedliskowych lasu i projektowania składów docelowych drzewostanów w obrębie gleb bielicoziemnych. Sylwan 143(5), 65-72.

Błońska, E., Lasota, J., Januszek, K., 2013. Variability of enzymatic activity in forest Cambisols and Brunic Arenosols of Polish lowland areas. Soil Science Annual 64(2), 54-59.

Brożek, S., Zwydak, M., 2010. Atlas gleb leśnych Polski. Centrum Informacyjne Lasów Państwowych, Warszawa. (In Polish with English summary)

Chojnicki, J., Kwasowski, W., Wójcik, R., 2021. Ocena funkcji i przeznaczenia Glebowej Powierzchni Wzorcowej w Puszczy Białej w zależności od typologii i właściwości gleb. Sylwan 165(3), 223-232. (In Polish with English summary)

Cornell, R.M., Schwertmann, U., 1979. The influence of organic anions on the crystallization of ferrihydrite. Clays and Clay Minerals 27, 402410. https://doi.org/10.1346/CCMN.1979.0270602

Czubaszek, R., Banaszuk, H., 2004. Wybrane właściwości gleb rdzawych na wydmach śródtorfowych w bagiennych dolinach Biebrzy i Narwi. Roczniki Gleboznawcze - Soil Science Annual 55(1), 87-98. (In Polish with English summary)

Degórski, M., 2002. Przestrzenna zmienność właściwości gleb bielicoziemnych środkowej i północnej Europy a geograficzne zróżnicowanie czynników pedogenicznych. Prace Geograficzne nr 182, PAN, IGiPZ, Warszawa. (In Polish with English summary)

Dłużewski, P., Wiatrowska, K., Kozłowski, M., 2019. Seasonal changes in organic carbon content in post-arable forest soils. Soil Science Annual 70(1), 3-12. https://doi.org/10.2478/ssa-2019-0001

FAO, 2006. Guidelines for Soil Description. FAO, Rome.

Ferczyńska-Uggla, Z., 1976. Związek między pokrywą glebową a niektórymi zbiorowiskami roślinnymi rezerwatu Borki w Puszczy Boreckiej. Roczniki Gleboznawcze - Soil Science Annual 27(1), 147-209. (In Polish with English summary)
Florek, W., 1991. Postglacjalny rozwój dolin rzek środkowej części północnego skłonu Pomorza. WSP, Słupsk. (In Polish with English summary)

Florinsky, I.V., Kuryakova, G.A., 1996. Influence of topography on some vegetation cover properties. Catena 27(2), 123-141. https://doi. org/10.1016/0341-8162(96)00005-7

Gonet, S.S., 2010. Wpływ pożaru lasu na właściwości materii organicznej gleb. (In:) Sewerniak, P., Gonet S.S. (Eds.) Środowiskowe skutki pożaru lasu. PTSH, Wrocław. (In Polish with English summary)

Hirsch, F., Schneider, A., Bauriegel, A., Raab, A., Raab, T., 2018. Formation, classification, and properties of soils at two relict charcoal hearth sites in Brandenburg, Germany. Frontiers in Environmental Science 6, 94. https://doi.org/10.3389/fenvs.2018.00094

IUSS Working Group WRB, 2015. World Reference Base for Soil Resources 2014, update 2015. International soil classification system for naming soils and creating legends for soil maps. World Soil Resources Reports No. 106. FAO, Rome.

Jankowski, M., 2014a. Bielicowanie jako wtórny proces w glebach rdzawych Brodnickiego Parku Krajobrazowego. (In:) Świtoniak M., Jankowski M., Bednarek R. (Eds.). Antropogeniczne przekształcenia pokrywy glebowej Brodnickiego Parku Krajobrazowego. Wydawnictwo UMK, Torun. (In Polish with English summary)

Jankowski, M., 2014b. The evidence of lateral podsolization in sandy soils of Northern Poland. Catena 112, 139-147. https://doi.org/10.1016/ j.catena.2013.03.013

Jankowski, M., 2019. Pokrywa glebowa. (in:) Sewerniak P., Holc J. Przyroda poligonu toruńskiego. Stan badań i problemy ochrony. Wydawnictwo Naukowe UMK, Toruń. (In Polish with English summary)

Janowska, E., 1994. Preliminary study on the sideric horizon of rusty soils with the use of microchemical x-ray analysis. Roczniki Gleboznawcze - Soil Science Annual 44, 41-53. (In Polish with English summary)

Janowska, E., 2001. Geneza i właściwości gleb rdzawych na obszarze zlodowacenia środkowopolskiego. Fundacja Rozwój SGGW, Warszawa. (In Polish with English summary)

Jenny, H., 1941. Factors of Soil Formation. A System of Quantitative Pedology. McGraw Hill, New York.

Jonczak, J., Olszak, I., Łazarczyk, A., 2013. Geneza, ewolucja i właściwości gleb niższej terasy nadzalewowej Słupi w południowej części Słupska In: Jonczak, J., Florek, W. (Eds.), Środowisko glebotwórcze i gleby dolin rzecznych. Wydawnictwo Naukowe Bogucki, Poznań, 57-66. (In Polish with English summary)

Kirschenstein, M., Baranowski, D., 2008. Wahania roczne i tendencje zmian opadów atmosferycznych i temperatury w Słupsku, Dokumentacja Geograficzna 37, 76-82. (In Polish with English summary)

Konecka-Betley, K., 1968. Zagadnienie żelaza w procesie glebotwórczym. Roczniki Gleboznawcze - Soil Science Annual 19(1), 51-97. (In Polish with English summary)

Konecka-Betley, K., 1977. Soils of dune areas of Central Poland in late glacial and Holocene. Folia Quaternalia 49, 41-58.

Konecka-Betley, K., Czępińska-Kamińska, D., Janowska, E., 1994. Właściwości fizykochemiczne i chemiczne gleb w Kampinoskim Parku Narodowym (stan na rok 1991). In: Konecka-Betley K. (Ed.) Prognozowanie przemian właściwości chemicznych gleb Kampinoskiego Parku Narodowego na tle innych komponentów środowiska przyrodniczego. Fundacja „Rozwój SGGW”, Warszawa. (In Polish with English summary)

Kowalkowski, A., 1977a. Dynamika rozwoju późnoplejstoceńskich i holoceńskich gleb z piasków wydmowych w Pomorsku. Roczniki Gleboznawcze - Soil Science Annual 28(3/4), 19-35. (In Polish with English summary)

Kowalkowski, A., 1977b. Wpływ różnej głębokości wody gruntowej na wilgotność i zasobność gleb rdzawych bielicowych pod drzewostanami sosnowymi. Roczniki Gleboznawcze - Soil Science Annual 28(3/4), 127-135. (In Polish with English summary)

Kowalkowski, A., Nowak, G., 1968. Gleby bielicowe Wzgórz Ostrzeszowskich wytworzone z pisaków akumulacji peryglacjalnej. Cz. 1. Wa- 
runki środowiska glebotwórczego. Roczniki Gleboznawcze - Soil Science Annual 19(1), 27-49. (In Polish with English summary)

Kozarski, S., 1995. Deglacjacja północno-zachodniej Polski: warunki środowiska i transformacja geosystemu ( 20 ka $\rightarrow \sim 10 \mathrm{ka} \mathrm{BP})$. Dokumentacja Geograficzna 1, IGiPZ PAN, Warszawa. (In Polish with English summary)

Kuźnicki, F., Białousz, S., Rusiecka, D., Skłodowski, P., 1974. Charakterystyka procesu bielicowania $\mathrm{w}$ glebach wytworzonych z piasków wydmowych Puszczy Kampinoskiej. Roczniki Gleboznawcze - Soil Science Annual 25(2), 25-51. (In Polish with English summary)

Kuźnicki F., Skłodowski P., 1974. Content of various forms of humus compounds in podzolized rusty soils and podzol, developed from fluvioglacial sand. Roczniki Gleboznawcze - Soil Science Annual 25, dodatek, 185-196. (In Polish with English summary)

Łabęda, D., Kondras, M., 2020. Influence of forest management on soil organic carbon stocks. Soil Science Annual 71(2), 165-173. https://doi. org/10.1016/j.foreco.2020.118127

Macyk, T.M., Pawluk, S., Lindsay, J.D., 1978. Relief and microclimate as related to soil properties. Canadian Journal of Soil Science 58, 421-438. https://doi.org/10.4141/cjss78-049

Malczyk, P., 1988. Formy żelaza w rdzawych glebach leśnych wytworzonych z piasku wydmowego. Roczniki Gleboznawcze - Soil Science Annual 39(3), 233-235. (In Polish with English summary)

Manikowska, B., 1997. Peryglacjalne utwory pokrywowe i kształtowanie profilu glebowego na wysoczyźnie fluwioglacjalnej w Polsce Środkowej. Roczniki Gleboznawcze - Soil Science Annual 48(3/4), 119-133. (In Polish with English summary)

Mehra, O., Jackson, J., 1960. Iron oxide removal from soils and clays by a dithionite-citrate system buffered with sodium bicarbonate. Clays and Clay Minerals 5, 317-327. https://doi.org/10.1016/B978-0-08009235-5.50026-7

Moeslund, J.E., Arge, L., Bøcher, P.K., Dalgaard, T., Odgaard, M.V., Nygaard, B., Svenning, J.C., 2013. Topographically controlled soil moisture is the primary driver of local vegetation patterns across a lowland region. Ecosphere 4(7), 91. http://dx.doi.org/10.1890/ES13-00134.1

Okołowicz, M., Czępińska-Kamińska, D., Janowska, E., Konecka-Betley, K., 2003. Rozmieszczenie fosforu w glebach rezerwatu biosfery „Puszcza Kampinoska”. Roczniki Gleboznawcze - Soil Science Annual 54(3), 39-48. (In Polish with English summary)

Pokojska, U., 1979. Geochemical studies on podsolization. Part I. Podzolization in the light of the profile distribution of various forms of iron and aluminium. Roczniki Gleboznawcze - Soil Science Annual 30(1), 189-215. (In Polish with English summary)

Prusinkiewicz, Z., 1965. Ustalenie wieku chronosekwencji glebowej na mierzejach Bramy Świny metodą radiowęgla ${ }^{14} \mathrm{C}$. Roczniki Gleboznawcze - Soil Science Annual, dodatek do tomu 15, 443-446. (In Polish with English summary)

PTG., 2009. Klasyfikacja uziarnienia gleb i utworów mineralnych - PTG 2008, Roczniki Gleboznawcze - Soil Science Annual 60(2), 5-17.

Sewerniak, P., Fifielska, D., Bednarek, R., 2014. Przekształcenia morfologii i właściwości gleb na skutek zabiegów przygotowujących glebę do odnowienia drzewostanu. In: Świtoniak M., Jankowski M., Bednarek R. (Eds.). Antropogeniczne przekształcenia pokrywy glebowej Brodnickiego Parku Krajobrazowego. Wydawnictwo UMK, Toruń. (In Polish with English summary)

Skłodowski, P., Maciejewska, A., Szafranek, A., 1988. Wpływ procesu bielicowania na rozmieszczenie pierwiastków śladowych w profilach gleb bielicowych. Roczniki Gleboznawcze - Soil Science Annual 39(4), 113-128. (In Polish with English summary)

Sommer, M., Halm, D., Weller, U., Zarei, M., Stahr, K., 2000. Lateral podzolization in a granite landscape. Soil Science Society of America Journal 64, 1434-1442. https://doi.org/10.2136/sssaj2000.6462069x

Świtoniak, M., 2006. Litologiczne uwarunkowania kierunku rozwoju procesów glebotwórczych w glebach o dwudzielnym uziarnieniu na terenie Pojezierza Brodnickiego. Dokumentacja Geograficzna 32, 278-285. (In Polish with English summary)

Van Reeuwijk, L., 1995. Procedures for soil analysis. Technical Paper 9, International Soil Reference and Information Centre.

\section{Słowa kluczowe}

Gleby rdzawe

Geneza gleb

Procesy stokowe

Krajobraz fluwioglacjalny

Właściwości gleb

\section{Przestrzenne zróżnicowanie gleb rdzawych i gleb towarzyszących wzdłuż stoku doliny Słupi (Pomorze Środkowe, północna Polska)}

\section{Streszczenie}

Badania miały na celu ocenę przestrzennego zróżnicowania pokrywy glebowej w obrębie fluwioglacjalnej toposekwencji na stoku doliny Słupi w okolicach Słupska. Procesy glebotwórcze i właściwości gleb dyskutowano w kontekście pochodzenia materiałów macierzystych i ich transformacji w procesach post-sedymentacyjnych, a także roli rzeźby terenu jako czynnika determinującego przestrzenną zmienność gleb i osadów. W 10 profilach glebowych oznaczono szerokie spektrum właściwości stosując standardowe metody, m.in. uziarnienie, zawartość pierwiastków ogółem, wskaźniki stanu eko-chemicznego oraz wskaźniki zwietrzenia i zaawansowania procesów glebotwórczych. Uzyskane wyniki potwierdziły duże znaczenie litologii i rzeźby terenu jako czynników w rozwoju gleb i kształtowaniu ich przestrzennej zmienności. Głównym składnikiem pokrywy glebowej w badanej sekwencji były gleby rdzawe, które wykazywały zróżnicowanie morfologiczne odzwierciedlające wpływ przekształceń post-depozycyjnych osadów wskutek erozji wodnej, a także innych procesów typowych dla stoków. Wartości stosunku Ti:Zr sugerują jednak jedynie lokalne przekształcenia pierwotnych osadów. Mniejszą miąższością solum w obrębie badanego stoku charakteryzowały się gleby przykrawędziowej części wysoczyzny, górnych części stoków oraz strefy przejściowej pomiędzy stokami a dnem doliny Słupi w porównaniu z glebami dolnych części stoków i den dolinek erozyjnych. Obecność poziomów pochodzenia deluwialnego w niektórych lokalizacjach stanowi zapis procesów erozji w minionych czasach. Katenalne rozmieszczenie wolnych i amorficznych form Fe i Al wskazuje na śródglebowe przemieszczenie tych substancji wraz z wodami. Badane gleby silnie różnią się pod względem zawartości oraz zasobów węgla organicznego i azotu. Największe zasoby tych składników notowano w glebach wysoczyzny i podnóży stoków, zaś najmniejsze w obrębie środkowych partii stoków. Zawartości P, K, Ca i Mg były typowe dla gleb piaszczystych. Wszystkie gleby wykazywały typowe wartości pH i właściwości sorpcyjne, jednakże zmienność katenalna była w tym zakresie znacząca. 